\title{
Transition to Chaos in Random Neuronal Networks
}

\author{
Jonathan Kadmon ${ }^{*}$ \\ Racah Institute of Physics and the Edmond and Lily Safra Center for Brain Sciences, \\ The Hebrew University of Jerusalem, 9190401, Israel \\ Haim Sompolinsky \\ Racah Institute of Physics and the Edmond and Lily Safra Center for Brain Sciences, \\ The Hebrew University of Jerusalem, 9190401, Israel \\ and Center for Brain Science, Harvard University, Cambridge, Massachusetts 02138, USA
}

(Received 22 January 2015; revised manuscript received 27 August 2015; published 19 November 2015)

Firing patterns in the central nervous system often exhibit strong temporal irregularity and considerable heterogeneity in time-averaged response properties. Previous studies suggested that these properties are the outcome of the intrinsic chaotic dynamics of the neural circuits. Indeed, simplified rate-based neuronal networks with synaptic connections drawn from Gaussian distribution and sigmoidal nonlinearity are known to exhibit chaotic dynamics when the synaptic gain (i.e., connection variance) is sufficiently large. In the limit of an infinitely large network, there is a sharp transition from a fixed point to chaos, as the synaptic gain reaches a critical value. Near the onset, chaotic fluctuations are slow, analogous to the ubiquitous, slow irregular fluctuations observed in the firing rates of many cortical circuits. However, the existence of a transition from a fixed point to chaos in neuronal circuit models with more realistic architectures and firing dynamics has not been established. In this work, we investigate rate-based dynamics of neuronal circuits composed of several subpopulations with randomly diluted connections. Nonzero connections are either positive for excitatory neurons or negative for inhibitory ones, while single neuron output is strictly positive with output rates rising as a power law above threshold, in line with known constraints in many biological systems. Using dynamic mean field theory, we find the phase diagram depicting the regimes of stable fixed-point, unstable-dynamic, and chaotic-rate fluctuations. We focus on the latter and characterize the properties of systems near this transition. We show that dilute excitatoryinhibitory architectures exhibit the same onset to chaos as the single population with Gaussian connectivity. In these architectures, the large mean excitatory and inhibitory inputs dynamically balance each other, amplifying the effect of the residual fluctuations. Importantly, the existence of a transition to chaos and its critical properties depend on the shape of the single-neuron nonlinear input-output transfer function, near firing threshold. In particular, for nonlinear transfer functions with a sharp rise near threshold, the transition to chaos disappears in the limit of a large network; instead, the system exhibits chaotic fluctuations even for small synaptic gain. Finally, we investigate transition to chaos in network models with spiking dynamics. We show that when synaptic time constants are slow relative to the mean inverse firing rates, the network undergoes a transition from fast spiking fluctuations with constant rates to a state where the firing rates exhibit chaotic fluctuations, similar to the transition predicted by rate-based dynamics. Systems with finite synaptic time constants and firing rates exhibit a smooth transition from a regime dominated by stationary firing rates to a regime of slow rate fluctuations. This smooth crossover obeys scaling properties, similar to crossover phenomena in statistical mechanics. The theoretical results are supported by computer simulations of several neuronal architectures and dynamics. Consequences for cortical circuit dynamics are discussed. These results advance our understanding of the properties of intrinsic dynamics in realistic neuronal networks and their functional consequences.

DOI: 10.1103/PhysRevX.5.041030

\footnotetext{
* Corresponding author. jonathan.kadmon@mail.huji.ac.il
}

Published by the American Physical Society under the terms of the Creative Commons Attribution 3.0 License. Further distribution of this work must maintain attribution to the author(s) and the published article's title, journal citation, and DOI.
Subject Areas: Interdisciplinary Physics, Nonlinear Dynamics, Statistical Physics

\section{INTRODUCTION}

The firing patterns of circuits in the central nervous system often exhibit a high level of temporal irregularity. The effect can be seen by the interspike interval (ISI) distribution, which, except for a short refractory period, is similar to that of a Poisson process [1-3]. Intracellular 
recordings [4] indicate that this irregularity is due to fluctuations in the synaptic input to the neurons, suggesting a dynamic origin, and motivating the exploration of underlying neuronal circuit mechanisms. A possibly related issue is the ubiquitous diversity of the time-averaged response properties of single neurons, e.g., their firing rates and tuning modulations, within a local population $[1,5]$.

Several theoretical studies explored the emergence of temporal irregularity and, in particular, chaotic dynamics in neuronal networks. These investigations focused on two types of models: rate-based models with Gaussian connections and spiking dynamics of sparsely connected excitatory-inhibitory networks. The first class uses a firing-rate dynamics in which each unit is characterized by a smooth function that maps the synaptic input into an output firing rate. In its simplest version, the input-output transfer function is $\tanh (x)$, where a zero value denotes some reference activity level and 1 and -1 denote saturated firing and quiescent states, respectively. The architecture of the rate model was given by a random connectivity matrix where each connection is drawn from a Gaussian distribution, with zero mean and variance given by $g^{2} / N, N$ being the size of the network. It was shown that the system exhibits a transition from a stable zero fixed-point state for low values of $g$ to a chaotic state for large $g$. Furthermore, for large $N$ this transition is sharp and occurs at $g_{c}=1$ [6]. In these models, the emergence of chaos is gradual, as the amplitude of the fluctuations, their inverse time constant, and the Lyapunov exponent vanish as $g \rightarrow 1^{+}$. The chaotic state is asynchronous in that the correlations between fluctuations of different neurons are weak (and vanish as $N \rightarrow \infty$ ).

The second class is motivated by biological reality. To capture the spiking dynamics, these models use either binary $\{0,1\}$ neurons or integrate-and-fire spiking neurons, and the connectivity is characterized by randomly sparse connections, where the mean number of connections, $K$, is much smaller than $N$. To capture the biological constraints on the sign of the connections, the networks consist of excitatory and inhibitory populations, where the nonzero output connections of excitatory (inhibitory) neurons are positive (negative). The dynamics is dominated by the competition between strong excitatory and inhibitory connections, leading to a dynamic cancellation of the excitatory and inhibitory inputs. The ensuing balanced state exhibits intrinsically generated Poisson-like stochasticity as well as asynchrony [7-10]. There is no parameter regime where the state can be characterized as a stable fixed point, and chaos does not emerge gradually as a function of synaptic strength. Instead, temporal irregularity is always strong, and correlation times are short. The origin of the qualitative difference in the behavior of the two types of models was never investigated comprehensively. In particular, it was unclear whether the differences originate from the different dynamics (a smooth rate dynamics vs spiking dynamics) or whether it is attributed to the different architectures: Gaussian-distributed synapses in a single, fully connected, and statistically homogeneous population (with mixed excitation and inhibition) vs a two-population (excitatory and inhibitory) architecture with sparse connectivity.

The question of the existence of a bifurcation to chaos is not only interesting from a dynamical-systems perspective but may also have important functional consequences. Several studies have highlighted the computational utility of the nonlinear dynamics of random, recurrent networks near the onset of chaos. For instance, a novel "reservoir computing" model has been proposed, which utilizes the rich intrinsic network dynamics to learn to generate complex temporal trajectories [11-17]. Reservoir computing is most effective above but near the transition to chaos because of the emergence of slow dynamical fluctuations since many applications involve dynamics with time scales of seconds, much larger than the microscopic time scales of a few milliseconds. Additionally, it has been shown that decoding signals from these networks is particularly robust above and near the transition to chaos [18,19]. A recent study by Saxe et al. [20] studies the dynamics of learning in deep networks. They define a critical point above which infinitely deep networks exhibit chaotic percolating activity propagation, analogous to the chaotic state of recurrent networks. Finally, recent advances in machine learning have generated resurgence of interest in recurrent networks (primarily of speech and language processing; see, e.g., Refs. [21-23] and references therein). Understanding the dynamics of generic recurrent networks will gain insight into these highly interesting computational capacities.

In this work, we study the existence and the properties of the transition to chaotic dynamics in a broad range of models that span the two model classes mentioned above. In Sec. II, we introduce a general architecture for random recurrent networks with multiple subpopulations, obeying smooth rate-based dynamics. We show the correspondence between randomly diluted networks in the balanced state and networks with Gaussian-distributed connections with the same multiple population architecture. Section III introduces the mathematical framework of the dynamic mean field theory (DMFT) used in analytical investigation of the properties of the network state and extends the theory of transition from fixed point to chaos, previously derived for a single Gaussian population to the more general architecture. In Sec. V, we apply the theory to the simple case of a single inhibitory neuronal population and a threshold-linear-synaptic transfer function. The example of a two-population model is studied in Sec. VI. Although the DMFT is more complex than the single-population network, we show that the two-population network exhibits a transition from fixed point to chaos that is similar to that of the single-population case. The role of the single-neuron nonlinear transfer function is elucidated in Sec. VII. First, 
we show that for a sufficiently sharp function, chaos exists in large systems at all gain values. Second, the shape of this function determines the critical behavior of the relaxation times and largest Lyapunov exponent near the transition.

Unlike the rate-based smooth dynamics, in models with spiking dynamics, a fixed-point state does not exist as long as some of the neurons are firing. Nevertheless, it is interesting to explore the conditions under which the spiking network exhibits a transition from a state with stationary inputs and firing rates to a state where the underlying inputs and rates fluctuate in time. In Sec. VIII, we use a Poisson spiking model to show that in the case where the synaptic integration time is much larger than the inverse of the neuronal firing rates, the synaptic current exhibits a sharp transition from a fixedpoint to chaotic dynamics similar to that of rate dynamics. The behavior for large but finite synaptic integration time is analyzed using scaling analysis, similar to that of a secondorder phase transition. The implications of the results for the understanding of the dynamics and computations in cortical circuits are discussed in Sec. IX.

\section{MODEL}

\section{A. Randomly diluted network with multiple populations}

We consider a network of neurons composed of $P$ subpopulations that are assumed, for convenience, to have equal size, $N$ [Fig. 1(a)]. The recurrent connections $J_{k l}^{i j}$ denote the synaptic efficacy between the presynaptic $j$ th neuron of the $l$ th population to the post synaptic $i$ th neuron of the $k$ th population, where $k, l=1, \ldots, P$, and $i, j=1, \ldots, N$. The connectivity is randomly diluted so that each connection $J_{k l}^{i j}$ is nonzero with probability $p$, where

$$
p=K / N
$$

and zero otherwise. Thus, the mean number of inputs to each neuron is $K \times P$. Some of the populations are assumed to be inhibitory. For an inhibitory population $l$, all $J_{k l}^{i j}$ are nonpositive. Conversely, all nonzero outgoing connections of an excitatory population are positive. In addition, each neuron from the $k$ th population receives a constant, uniform input, equal to $m_{0} W_{k}$, where the parameter $m_{0}$ denotes the mean activity (firing rate) of neurons in the input population, and $W_{k}$ are assumed positive.

Throughout this work, we focus on networks with a high degree of connectivity, i.e., $N, K \gg 1$. Although in most previous analytical work on the dynamics of dilute neuronal networks it was assumed that the network connectivity is sparse (i.e., $1 \ll K \ll N$ ) $[8,10,24,25]$, here we assume only that $1 \ll K<N$, allowing for a dense regime as well. (In Refs. [9,26], densely connected networks were studied,
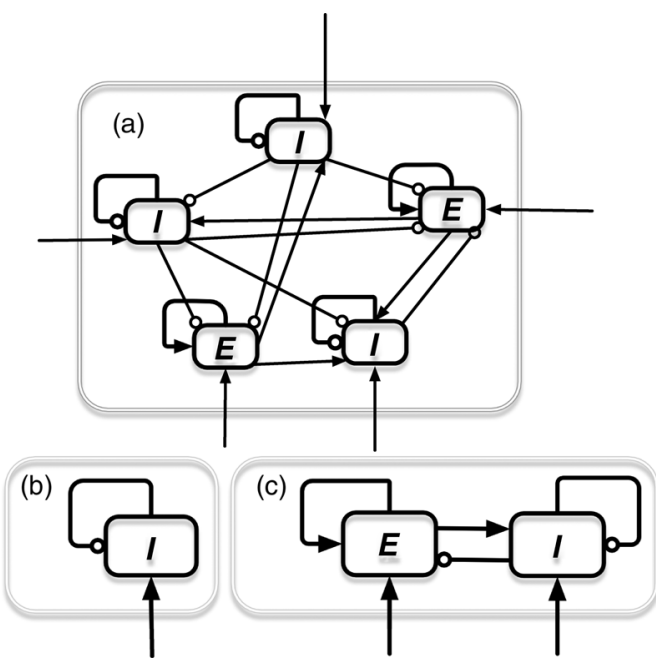

FIG. 1. Network schematics. Arrows (circles) denote excitatory (inhibitory) connections. (a) The general network architecture studied here consists of multiple excitatory $(E)$ and inhibitory $(I)$ subpopulations driven by external inputs. Individual connections are randomly diluted or are Gaussian distributed. Subpopulations are distinguishable by the different statistics of connectivity. (b) The simplest model consists of a single population with random inhibitory recurrent connections with an external excitatory input. (c) Two randomly connected $E$ and $I$ populations.

but the focus was on the spatial patterns and correlations. The dynamical properties and the temporal autocorrelations were not addressed.) In order for the dynamics to be affected by the fluctuations in connectivity, we assume all nonzero connections equal $J_{k l} / \sqrt{K}$, as discussed below. Similarly, the external connections scale as $W_{k}=\omega_{k} \sqrt{K}$.

\section{B. Rate dynamics}

We first study a firing rate model for the dynamics of neuronal activity. The state of each neuron, say, with indices $(i, k)$, at time $t$, is given by its total synaptic current, $h_{k}^{i}(t)$, which obeys a first-order nonlinear dynamics,

$$
\frac{d}{d t} h_{k}^{i}(t)=-h_{k}^{i}(t)+\sum_{l=1}^{P} \sum_{j=1}^{N} J_{k l}^{i j} \phi\left(h_{l}^{j}(t)\right)+W_{k} m_{0} .
$$

The first term is a decay term, the second is the synaptic input from the network, and the last is the synaptic input from the external source. For convenience, we set all synaptic time constants to unity. The nonlinear transfer function $\phi(h)$ denotes the firing rate of a neuron with an input synaptic current $h$ and is analogous to the neuronal input-current to firing-rate transfer function, known as the $f$-I curve. 


\section{Effective Gaussian connectivity}

Past work on random highly connected systems has shown that under fairly general conditions, in the limit of large $K$, the system's behavior depends only on the first two moments of the connectivity matrix [27,28]. Hence, the connectivity matrix $J_{k l}^{i j}$ can be replaced by a random matrix of Gaussian-distributed connections where the mean and variance are matched to that of the dilute network. We thus consider a general dynamics of a multiple population network with a fully connected connectivity matrix with Gaussian-distributed connections, $J_{k l}^{i j}=\left(\bar{g}_{k l} / N\right)+\mathcal{J}_{k l}^{i j}$,

$$
\begin{aligned}
\frac{d}{d t} h_{k}^{i}(t)= & -h_{k}^{i}(t)+\sum_{l=1}^{P} \sum_{j=1}^{N} \mathcal{J}_{k l}^{i j} \phi\left(h_{l}^{j}(t)\right) \\
& +\sum_{l=1}^{P} \bar{g}_{k l} m_{l}(t)+h_{k}^{0},
\end{aligned}
$$

where

$$
m_{k}(t)=\frac{1}{N} \sum_{i=1}^{N} \phi\left(h_{k}^{i}(t)\right)
$$

are the mean population activities. The coefficients $\mathcal{J}_{k l}^{i j}$ are quenched Gaussian variables with zero mean and variance,

$$
\left\langle\left(\mathcal{J}_{k l}^{i j}\right)^{2}\right\rangle=\frac{g_{k l}^{2}}{N} .
$$

We refer to $g$ as the gain of the synaptic input. The contribution of the mean connections, $\bar{g}_{k l} / N$, is represented by the third term in the right-hand side of Eq. (3).

In the dilute network, the mean connection between two populations equals $p J_{k l} / \sqrt{K}=\sqrt{K} J_{k l} / N$. The variances of these connections are $p(1-p) J_{k l}^{2} / K=(1-K / N) J_{k l}^{2} / N$. Hence, the corresponding parameters of the equivalent Gaussian network are

$g_{k l}^{2}=(1-K / N) J_{k l}^{2}, \quad \bar{g}_{k l}=\sqrt{K} J_{k l}, \quad h_{k}^{0}=\sqrt{K} \omega_{k} m_{0}$.

Note that in the dilute networks, $g_{k l}$ and $\bar{g}_{k l}$ are related through

$$
\bar{g}_{k l}=g_{k l} \sqrt{\frac{K}{(1-K / N)}} .
$$

In the theory below, we analyze the dynamics of the Gaussian network, defined by Eq. (3), in its generality; namely, we consider the parameters describing the mean and the variance of the connections, $\bar{g}_{k l}$ and $g_{k l}$, as independent parameters. In addition, we will not restrict ourselves to the case in which the mean connections $\bar{g}_{k l}$ and $h_{k}^{0}$ are large. The relation Eq. (7), as well as the scaling of $\bar{g}_{k l}$ and $h_{k}^{0}$ with $O(\sqrt{K})$, will be adopted when applying the theory to the balanced dilute networks. The numerical simulations will use both Gaussian and random dilution and will show their equivalence.

\section{Balanced regime}

In the diluted network model, the net input from the $l$ th population to each of the $k$ th neurons is proportional to $\bar{g}_{k l}$, and $h_{k}^{0}$ hence scales as $\sqrt{K}$. On the other hand, the fluctuations scale as $g_{k l}$, and hence are of order 1. Thus, it would seem that whenever the degree of connectivity, $K$, is high, the fluctuations induced by the random dilution will have a negligible effect on the dynamics, reducing the system to that with uniform connections and anomalously strong recurrent and external inputs. In fact, the system avoids this saturated state by dynamically canceling the mean excitatory contributions against the inhibitory ones, resulting in the net mean inputs, which are of order 1, and of the same order as the fluctuations. Balanced states in networks with strong excitatory and inhibitory connections were previously studied (see Refs. [8,29]) in the context of binary or spiking networks. As we will show below, networks of units with smooth rate-based dynamics and transfer functions with finite gains also settle in this balanced state.

\section{FIXED POINTS AND THEIR STABILITY}

\section{A. Mean field equations for the fixed points}

To fully characterize the state of the system, we separate the population-averaged quantities, which are time independent in the stationary state, from the fluctuating ones, writing

$$
h_{k}^{i}(t)=u_{k}+\delta h_{k}^{i}(t)
$$

where

$$
u_{k}=\sum_{l} \bar{g}_{k l} m_{l}+h_{k}^{0} .
$$

The fluctuating inputs obey

$$
\frac{d}{d t} \delta h_{k}^{i}(t)=-\delta h_{k}^{i}(t)+\eta_{k}^{i}(t),
$$

where the spatiotemporal fluctuations in the currents, $\delta h_{k}^{i}(t)$, are low-pass temporal filters of the fluctuating synaptic inputs,

$$
\eta_{k}^{i}(t)=\sum_{l} \sum_{j} \mathcal{J}_{k l}^{i j} \phi\left(h_{l}^{j}(t)\right) .
$$

In the limit of a large number of inputs per neuron, these quantities obey Gaussian statistics with zero mean and zero 
spatial correlation but with temporal correlations that need to be evaluated self-consistently as explained below.

First, we study the fixed-point solution of the network dynamics, corresponding to a time-independent state, where $\delta h_{k}^{i}(t)=\delta h_{k}^{i}=\delta \eta_{k}^{i}$ are Gaussian distributed with zero mean and variance $\Delta_{k} \equiv\left\langle\left(\delta h_{k}^{i}\right)^{2}\right\rangle$. This quantity is calculated self-consistently as

$$
\Delta_{k}=\left\langle\left(\eta_{k}^{i}\right)^{2}\right\rangle=\sum_{l} g_{k l}^{2} C_{l},
$$

where $C_{l}=\left\langle\phi^{2}(h)\right\rangle$ are the average autocorrelations of the neuronal activities. Finally, using $h_{k}^{i}=u_{k}+\delta \eta_{k}^{i}$, we obtain the self-consistent equation for $\Delta_{k}$,

$$
C_{k}=\left\langle\phi^{2}\left(\sqrt{\Delta_{k}} z+u_{k}\right)\right\rangle .
$$

The constants $u_{k}$ are determined self-consistently, via Eq. (9), and

$$
m_{k}=\left\langle\phi\left(\sqrt{\Delta_{k}} z+u_{k}\right)\right\rangle .
$$

Note that in mean field theory, all averages denote integration over the Gaussian variables ( $z$ in the above equations), which have zero means and unit variance. A similar formulation, generalizing the stationary solution of a single-population mean field treatment to several populations, was recently employed by Cabana in Ref. [30].

\section{B. Balance equations}

In balanced architectures, both $\bar{g}_{k l}$ and $h_{k}^{0}$ are of order $\sqrt{K}$ [see Eq. (6)]. In this case, the self-consistent equations for $m_{k}$ assume a simple form. This is because, for the system to settle into an unsaturated state, $u_{k}$ must be of order 1; hence, Eq. (9) yields (to leading order in $\sqrt{K}$ )

$$
\sum_{l} \bar{g}_{k l} m_{l}+h_{k}^{0}=0, \quad \forall k .
$$

Since the mean rates are non-negative, Eq. (15) can be obeyed only for a range of $\bar{g}_{k l}$ and $h_{k}^{0}$ values. Stability of the balanced state (see Ref. [8]) further restricts the parameter regimes. We refer to these restrictions on the parameters as the balance conditions. Substituting the solution to the balance equations into Eq. (14) yields equations for the residual, order-1 mean inputs, $u_{k}$.

\section{Stability of fixed points}

Stability of the population-averaged activities.-The stability equations for the population-averaged degrees of freedom are determined by considering the response of the system to perturbations in the external fields, $\delta h_{k}^{0}$, which are uniform within the populations. It is convenient to define the uniform linear response by

$$
\chi_{k l}(t) \equiv \frac{\partial m_{k}(t)}{\partial h_{l}(0)}=\frac{1}{N} \sum_{i}^{N} \partial \phi_{k}^{i}(t) / \partial h_{l}^{0}(0) .
$$

Note that due to the spatial summation, this quantity is essentially averaged over $\mathcal{J}$. Interestingly, the response of the population-averaged activity is coupled to the response of the population variances, defined as

$$
\chi_{k l}^{\Delta}(t)=\frac{\partial}{\partial h_{l}^{0}(0)} \Delta_{k}(t)=\frac{\partial}{\partial h_{l}^{0}(0)}\left\langle\delta h_{k}^{i}(t) \delta h_{k}^{i}(t)\right\rangle .
$$

In Appendix B, we derive the following coupled equations for the two sets of susceptibilities in the temporal Fourier domain,

$$
\left[\begin{array}{cc}
(\mathbf{I}-\mathbf{A} \overline{\mathbf{g}}+i \omega) & -\mathbf{B} \\
-\mathbf{E} \overline{\mathbf{g}} & (\mathbf{I}-\mathbf{D}+i \omega)
\end{array}\right]\left[\begin{array}{c}
\chi(\omega) \\
\chi^{\Delta}(\omega)
\end{array}\right]=\left[\begin{array}{l}
\mathbf{A} \\
\mathbf{E}
\end{array}\right] .
$$

Here, $\chi$ and $\chi^{\Delta}$ are both $P \times P(P$ being the number of populations). The $P \times P$-dimensional matrices appearing in Eq. (18) are defined as

$$
\begin{aligned}
A_{k l} & =g_{k l}^{2}\left\langle\phi_{l} \phi_{l}^{\prime}\right\rangle+\left\langle\phi_{l}^{\prime}\right\rangle, \\
B_{k l} & =\frac{1}{2} g_{k l}^{2}\left\langle\phi_{k}^{\prime \prime}\right\rangle\left[\left\langle\left(\phi_{l}^{\prime}\right)^{2}\right\rangle+\left\langle\phi_{l} \phi_{l}^{\prime \prime}\right\rangle\right], \\
D_{k l} & =g_{k l}^{2}\left(\left\langle\left(\phi_{l}^{\prime}\right)^{2}\right\rangle+\left\langle\phi_{l} \boldsymbol{\phi}_{l}^{\prime \prime}\right\rangle\right), \\
E_{k l} & =2 g_{k l}^{2}\left\langle\phi_{l} \phi_{l}{ }^{\prime}\right\rangle .
\end{aligned}
$$

Thus, the fixed point is stable against populationaveraged perturbations, provided that all the eigenvalues of the matrix

$$
\left[\begin{array}{cc}
\mathbf{I}-\overline{\mathbf{g}} \mathbf{A} & -\mathbf{B} \\
-\overline{\mathbf{g}} \mathbf{E} & \mathbf{I}-\mathbf{D}
\end{array}\right]
$$

have a negative real part.

Stability against local perturbations.-We now study the stability of the fixed point against small perturbations in the form of infinitesimal local fields $h_{k}^{0 i}$. It is convenient to define the local susceptibility matrix

$$
\chi_{k l}^{i j}(t)=\partial h_{k}^{i}(t) / \partial h_{l}^{j 0}(0) .
$$

The average of the off-diagonal elements of this matrix is zero, and their variance is $\mathcal{O}(1 / N)$; hence, we focus on the mean-square susceptibility matrix $\mathbf{G}$, defined in the Fourier domain by

$$
G_{k l}\left(\omega_{1}, \omega_{2}\right)=N^{-1} \sum_{i j}\left\langle\chi_{k l}^{i j}\left(\omega_{1}\right) \chi_{k l}^{i j}\left(\omega_{2}\right)\right\rangle .
$$

In Appendix $\mathbf{C}$, we show that the matrix $\mathbf{G}$ is

$$
\mathbf{G}\left(\omega_{1}, \omega_{2}\right)=\left[\left(1+i \omega_{1}\right)\left(1+i \omega_{2}\right) I-\mathbf{M}\right]^{-1},
$$


where

$$
M_{k l}=g_{k l}^{2}\left\langle\left(\phi^{\prime}\right)^{2}\right\rangle .
$$

Thus, the fixed points are stable, provided that the real parts of all eigenvalues of the $P \operatorname{dim}$ matrix $\mathbf{M}$ are less than 1 . In general, $\mathbf{M}$ is not symmetric; however, the largest (in absolute value) of the eigenvalues is real. We call $\mathbf{M}$ the stability matrix.

In conclusion, we have derived two stability conditions: one related to population-averaged perturbations, Eq. (19), and a second related to local perturbations, associated with Eq. (22). Note that the mean interactions $\bar{g}$ do not appear in the latter condition. This is because the contribution to the off-diagonal elements of Eq. (21) from the uniform susceptibility is only of the order $1 / N$. For the fixed point to be stable, both conditions must hold. However, the instability associated with each condition has a different implication. The population-averaged instability signals either a runaway (as we show in a concrete example in Sec. V) or a transition to another stable fixed point, a stable limit cycle, or some other coherent spatiotemporal states. On the other hand, as we show below, the instability in Eq. (22) signals a transition to an asynchronous chaotic state.

Which of the instabilities occurs first when one varies one of the parameters depends on the specific architecture and parameter sets. Specific examples will be shown below.

\section{CHAOTIC STATE: DYNAMIC MEAN FIELD THEORY}

The chaotic state is an asynchronous state with stationary statistics, governed by the local-field autocorrelation functions

$$
\Delta_{k}(\tau) \equiv\left\langle\delta h_{k}^{i}(t) \delta h_{k}^{i}(t+\tau)\right\rangle
$$

where the angular brackets denote both average over neurons in the population and over time $t$. The self-consistent equations for these functions, derived from the dynamic mean field theory (DMFT) are (see Appendix A for a detailed derivation)

$$
\left(1-\frac{\partial^{2}}{\partial \tau^{2}}\right) \Delta_{k}(\tau)=\sum_{l} g_{k l}^{2} C_{l}(\tau),
$$

where $C_{k}(\tau)=\left\langle\phi\left(h_{k}^{i}(t)\right) \phi\left(h_{k}^{i}(t+\tau)\right)\right\rangle$ are the firing-rate autocorrelation functions, which depend on $\Delta_{k}(\tau)$ through

$C_{k}(\tau)=\left\langle\left\langle\phi\left(\sqrt{\Delta_{k}^{0}-\Delta_{k}(\tau)} y+\sqrt{\Delta_{k}(\tau)} z+u_{k}\right)\right\rangle_{y}^{2}\right\rangle_{z}$.

Here, both $y$ and $z$ are Gaussian-distributed random variables with zero means and unit variances. The boundary conditions for the solution are $\partial \Delta_{k}(0) / \partial \tau=0$, $\partial \Delta_{k}^{2}(\infty) / \partial \tau^{2}=0$, and $\Delta_{k}(0)=\Delta_{k}^{0}$. The first condition stems from the general fact that $\Delta(\tau)$ is a symmetric, continuous function. The second one comes from the requirement that $\Delta(\tau)$ converges to a finite value as $\tau \rightarrow \infty$ for a chaotic solution. The solution of Eq. (26) subject to these boundary conditions yields a unique solution for $\Delta_{k}(\tau)$ that converges to a fixed value, $\Delta_{k}(\infty)$, at long times. Except for networks with $h \rightarrow-h$ symmetry, the quantity $\Delta_{k}(\infty)$ is, in general, not zero, and it represents the variance of the Gaussian distribution of the time-averaged synaptic currents. The fluctuations in these inputs determine the fluctuations in the time-averaged firing rates of individual neurons.

Details of the derivation of the DMFT are given in Appendix A. Alternative formalisms for deriving dynamic mean field equations for such architectures include Faugeras and coworkers' use of McKean-Vaslov FokkerPlank formalisms [31] or stochastic networks via path integrals (see, e.g., Refs. [32,33]).

Lyapunov exponent.-In the chaotic state, we expect the squared susceptibility to show an exponential divergence with time, with a constant rate given by the largest Lyapunov exponent (LE). LE is defined as

$$
\lambda_{L}=\lim _{\tau \rightarrow \infty} \frac{1}{2 \tau} \ln \sum_{k l}\left(G_{k l}(\tau)\right),
$$

where

$$
G_{k l}(\tau)=\lim _{t \rightarrow \infty} \frac{1}{N} \sum_{i, j}^{N}\left(\frac{\partial h_{k}^{i}(t+\tau)}{\partial h_{l}^{0 j}(t)}\right)^{2} .
$$

Extending previous calculations [6], we show in Appendix $C$ that the long time behavior of $\mathbf{G}(\tau)$ is determined by the ground state of a quantum mechanical problem with the Hamiltonian operator defined as

$$
\mathcal{H}=-\frac{\partial^{2}}{\partial \tau^{2}} \mathbf{I}+\mathbf{I}-\mathbf{M}(\tau)
$$

where

$$
M_{k l}(\tau)=g_{k l}^{2}\left\langle\left\langle\phi^{\prime}\left(\sqrt{\Delta_{l}^{0}-\Delta_{l}(\tau)} y+\sqrt{\Delta(\tau)} z+u_{l}\right)\right\rangle_{y}^{2}\right\rangle_{z} .
$$

Finally, the LE is equal to

$$
\lambda_{L}=-1+\sqrt{1-\epsilon_{0}},
$$

where $\epsilon_{0}$ is the ground-state energy of $\mathcal{H}$. Note that, in general, $\mathcal{H}$ is not Hermitian. However, complex values of the Lyapunov exponents lead to unphysical oscillations of the squared susceptibility, so the ground-state energy is 
expected to be real and negative. A direct rigorous proof is still missing. In the regime of a stable fixed point, $\mathbf{M}$ is time independent and $\epsilon_{0}>0$, recovering the same stability criterion as above. A transition from a stable fixed point to chaotic dynamics occurs as $\epsilon_{0}$ vanishes.

\section{SINGLE INHIBITORY POPULATION WITH THRESHOLD-POWER-LAW TRANSFER FUNCTION}

Solving the DMFT for general networks requires extensive numerics. Many of the salient features are captured by the simplest case of a single inhibitory population [Fig. 1(b)] driven by a constant external excitatory input. The systems's dynamics,

$$
\frac{d}{d t} h^{i}(t)=-h^{i}(t)+\sum_{j=1}^{N} \mathcal{J}^{i j} \phi\left(h^{j}(t)\right)+\bar{g} m(t)+h^{0},
$$

is characterized by the recurrent inhibitory mean gain parameter $\bar{g}<0$, the gain parameter of the synaptic fluctuations, $g^{2}=N\left\langle\left(\mathcal{J}^{i j}\right)^{2}\right\rangle$, and the excitatory external input, $h^{0}>0$. The system is further simplified by assuming a transfer function of a threshold-power-law form $[34,35]$,

$$
\phi(x)=[x]_{+}^{\nu}, \quad \nu>0,
$$

where $[x]_{+}=\max (x, 0)$. For reasons explained below, we see that this monomial form is general enough when one studies the properties of the chaotic instability in networks with continuous threshold transfer functions.

\section{A. Fixed point and its stability}

In this model, the mean field equations for the fixed point are particularly simple. First, the mean input and its variance are given, respectively, by

$$
u=\bar{g} m+h^{0},
$$

where $m$ is the spatially averaged activity, $\left\langle\phi_{i}\right\rangle$, and

$$
\Delta=\left\langle\delta h_{i}^{2}\right\rangle=g^{2} C,
$$

where $\delta h_{i}=h_{i}-u$ and $C$ is the mean square activity, $\left\langle\phi_{i}^{2}\right\rangle$.

The mean field equations for $m$ and $C$ are given by

$$
m=\Delta^{\nu / 2}\left\langle[z+x]_{+}^{\nu}\right\rangle
$$

and

$$
C=\Delta^{\nu}\left\langle[z+x]_{+}^{2 \nu}\right\rangle
$$

Here,

$$
x=u / \sqrt{\Delta},
$$

representing the mean input in units of the standard deviation, and we have used the homogeneity property of the transfer function.

Substituting Eq. (37) into Eq. (35), the parameter $x$ can be determined from

$$
1=\tilde{g}^{2}\left\langle[z+x]_{+}^{2 \nu}\right\rangle,
$$

where $\tilde{g}=g \Delta^{(\nu-1) / 2}$. The values of $m$ and $\Delta$ are evaluated using Eqs. (34), (36), and (38).

As one increases $g$, the fixed point becomes unstable. Whether the first instability is the chaotic one [Eq. (22)] or the uniform one [Eq. (18)] will generally depend on the parameter set $\left(\nu, \bar{g}\right.$, and $\left.h^{0}\right)$.

Chaotic stability.-A transition to a chaotic state occurs at the fixed-point state when

$$
1=\tilde{g}^{2} \nu^{2}\left\langle[z+x]_{+}^{2(\nu-1)}\right\rangle .
$$

For a threshold-linear transfer function $(\nu=1), \tilde{g}=g$ is independent of $\Delta$, and critical values $g_{c}$ and $x_{c}$, at which a bifurcation from a fixed point to chaotic dynamics occurs, are determined by Eqs. (39) and (40) and are independent of $h^{0}$. In this case, $C^{\prime}(x)=H(-x)$ with $H(x)=$ $(1 / \sqrt{2 \pi}) \int_{x}^{\infty} d z e^{-\left(z^{2} / 2\right)}$, and transition occurs when $g_{c}=$ $\sqrt{2}$ and $x_{c}=0$. For nonlinear transfer functions, the critical values $x_{c}$ and $g_{c}$ depend on the values of $h^{0}$ and $\bar{g}$.

Uniform stability.-The stability condition for a network of a single population against uniform perturbations is given by [see Appendix B, where we derive the uniform stability condition in Eq. (18) for the case of a single population]

$$
g^{2}\left(\left\langle\phi^{\prime 2}\right\rangle+\left\langle\phi \phi^{\prime \prime}\right\rangle+\frac{\left\langle\phi^{\prime \prime}\right\rangle\left\langle\phi \phi^{\prime}\right\rangle \bar{g}}{\left(1-\left\langle\phi^{\prime}\right\rangle \bar{g}\right)}\right)<1 .
$$

For $\nu=1,\left\langle\phi \phi^{\prime \prime}\right\rangle$ vanishes, and the third term on the lefthand side of Eq. (41) is negative for $\bar{g}<0$; hence, a chaotic instability [Eq. (40)] always occurs first (i.e., for lower values of $g$ ). For $\nu \neq 1$, the occurrence of uniform stability of the fixed point will depend on the parameters $\bar{g}$ and $h^{0}$.

\section{B. Chaotic state}

In the threshold-linear model, it is useful to define the normalized autocorrelation

$$
q(\tau) \equiv 1-\Delta(\tau) / \Delta(0)
$$

as well as

$$
q(\infty)=1-\Delta(\infty) / \Delta(0)
$$


which measures the normalized variance of the dynamics, namely, the (spatially averaged) temporal variance of the local fields normalized by the squared amplitude. The autocorrelation function obeys Eq. (25),

$$
\left(1-\frac{\partial^{2}}{\partial \tau^{2}}\right) \Delta(\tau)=g^{2} C(\tau)
$$

Normalizing by $\Delta_{0}$ and using the homogeneity of the transfer function as above, we can define a Newtonian equation of motion on the normalized autocorrelation (42), which reads

$$
\frac{\partial^{2}}{\partial \tau^{2}} q(\tau)=-\frac{\partial V}{\partial q}
$$

The potential $V$ is given by

$$
\begin{aligned}
V(q) \equiv & -\frac{1}{2}(1-q)^{2} \\
& +\tilde{g}^{2} \int_{-\infty}^{\infty} D z\left[\int_{-\infty}^{\infty} D y \Phi(\sqrt{q} y+\sqrt{(1-q)} z+x)\right]^{2},
\end{aligned}
$$

where $\int D x=\int d x \exp \left(x^{2} / 2\right) / \sqrt{2 \pi}$ and $\Phi(h)=\int_{0}^{h} d y \phi(y)=$ $[h]_{+}^{\nu+1} /(\nu+1)[6]$. In the above equation, we have used the equality $\Delta_{0}=\Delta(0)$ and $\tilde{g} \equiv g \Delta_{0}^{(\nu-1) / 2}$. The initial conditions for Eq. (45) are $q(0)=0, \partial q(0) / \partial \tau=0$, and $\partial^{2} q(\infty) / \partial \tau^{2}=0$. The second condition stems from the general fact that $\Delta(\tau)$ is a symmetric, continuous function. The last one comes from the requirement that $\Delta(\tau)$ converges to a finite value as $\tau \rightarrow \infty$. These three conditions yield a unique solution and a unique value for the normalized mean input $x$.

Because of the existence of a potential, $x$ can be obtained without explicitly solving for $q(\tau)$ as follows. The above boundary conditions imply that the initial energy equals the potential energy $V(0)$, while the final energy equals the final potential energy $V(q(\infty))$. Thus, conservation of total energy yields $V(0)=V(q(\infty))$. Finally, for $q(\infty)$ to be an equilibrium point, the force must vanish; hence, $\partial V(q(\infty)) / \partial \tau=0$. These two equations determine both $q(\infty)$ and $x$. Once $x$ is known, Eq. (45) is integrated numerically to yield $q(\tau)$. Finally, $\Delta(\tau)$ is evaluated by solving the mean field equation (36) with $\Delta=\Delta_{0}$.

Stability of the chaotic solution.-The existence of a bounded chaotic phase, in which the mean activity of the network does not diverge and the trajectories of the local fields remain bounded, requires stability of the uniform mode. Unfortunately, a theory of uniform stability in a time-dependent dynamical state is lacking. However, in the linear case, one can find simple arguments for the existence of a chaotic solution. In this case, the normalized mean input $x<0$ in the chaotic phase is independent of $h^{0}$, and one finds that only when

$$
|\bar{g}|>\frac{|x|}{\left\langle[z+x]_{+}\right\rangle}
$$

does a solution for the mean field equation exist; smaller values of $|\bar{g}|$ entail dynamical instability.

For nonlinear transfer functions, the above argument does not hold. For $\nu=2$, for example, a solution for the MF equation exist for any value of $h^{0}$ and $\bar{g}$. However, numerical simulations show that an instability in the chaotic phase exists, as can be seen in Fig. 2 .

We note that this instability results from the unboundedness of $\phi$. For $\phi$ with a saturation level, the dynamics will always be bounded, but a crossover is expected from fluctuations spanning the linear dynamic range of the neurons when the net inhibition is large, to "epileptic" fluctuations in which neurons fluctuate between their saturated levels for weak inhibition.

Existence of a fixed-point solution.-An interesting result that is implied by Eq. (40) is that a stable fixed point (FP) exists only when

$$
\nu>1 / 2 \text {. }
$$

In contrast, when $\nu \leq 1 / 2$, the right-hand side of the FP stability condition, Eq. (40), diverges, indicating that no stable fixed-point solution exists for finite $g$, and depending on $g$, the system is either in a stable chaotic state or it diverges. This prediction is confirmed by the numerical simulations [see Fig. 2(a)] in which the normalized variance of the fields $q(\infty)$ is plotted as a function of $\nu$. For $\nu$ values of 0.5 or smaller, the system is in a chaotic state even for small values of $g$.

The instability of the fixed point for small $\nu$ is due to the presence of positive local fields that are arbitrarily close to zero. Thus, for a system of finite size, where the positive local fields are always of a nonzero minimum value, we expect that a fixed point will be stable at sufficiently small values of $g$. In the following sections, we focus on networks with threshold-linear $(\nu=1)$ and quadratic $(\nu=2)$ transfer functions, which exhibit a fixed point, a chaotic regime, and therefore a transition.

Finally, we note that the same arguments hold for the multiple population case as well.

\section{Phase diagram}

In Fig. 2(b), we show the phase diagram for the threshold-linear $(\nu=1)$, depicting the regimes of stable fixed point, chaos, and unstable dynamics in the parameter space of $g$ and $\bar{g}$. For values of $g<\sqrt{2}$, the network settles into a fixed point. For larger gain values, if the inhibition is strong enough, i.e., the condition in Eq. (47) holds, then the dynamics is chaotic and bounded to a finite regime in the state phase. For lower values of the uniform inhibition, the mean activity diverges. Note that because of the semilinearity of the transfer function, the phase diagram 

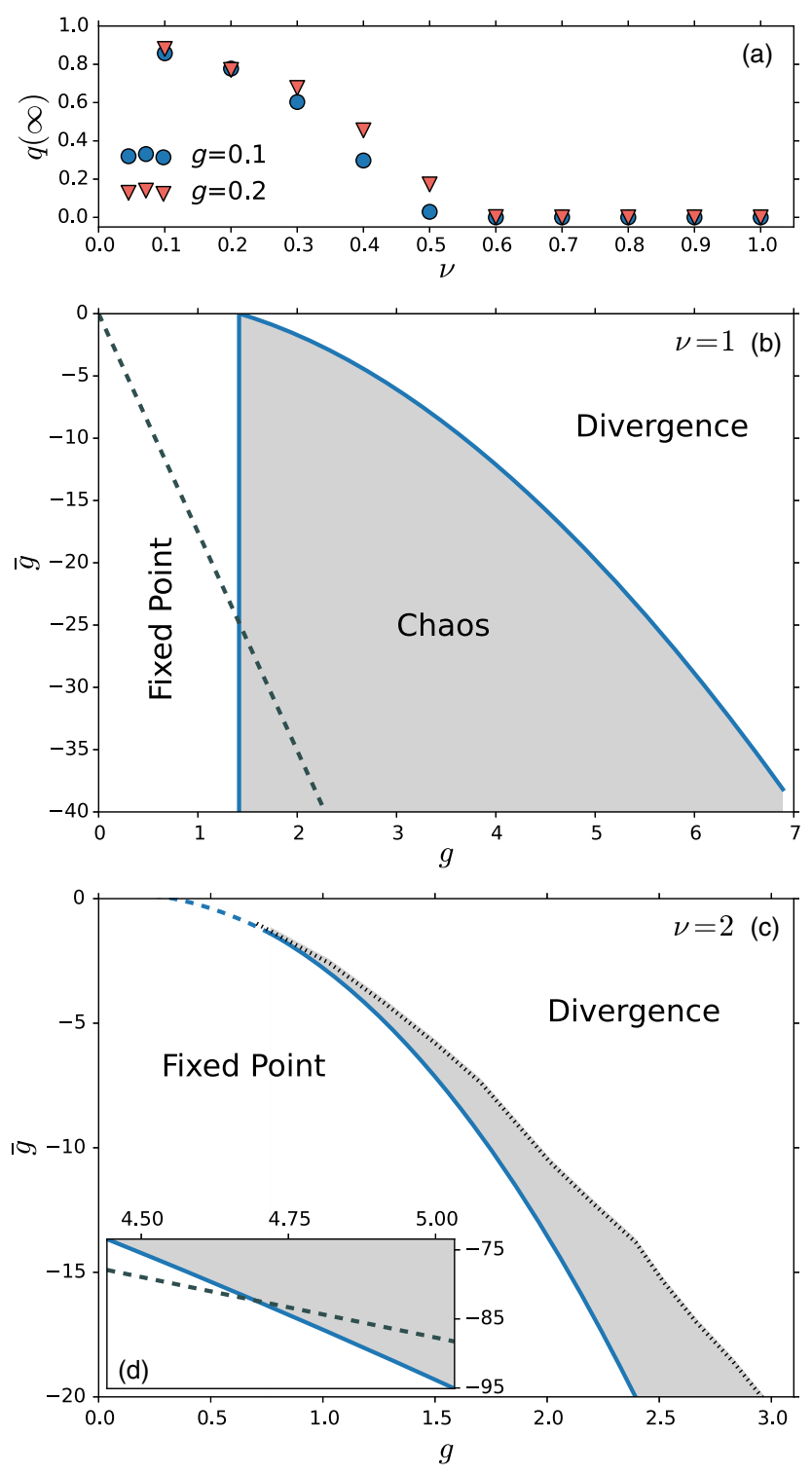

FIG. 2. Phase diagrams for a single inhibitory population. (a) No stable fixed point for sharp transfer functions. Simulation results for the normalized variance $q(\infty)$ for networks with $g=0.2$ and $g=0.1$ and different values of the power law, $\nu$, characterizing the rise of the transfer function, Eq. (33). Below $\nu=0.5$, the variance is nonzero, implying that there are temporal fluctuations even for low values of $g$. Simulation is performed on an inhibitory network $(N=5000)$ with randomly diluted connections and mean activity of $m=0.1$. (b) Phase-space diagram for a threshold-linear $(\nu=1)$ network of inhibitory neurons with mean connectivity $\bar{g}<0$ and variance $g$. The dashed line shows an example of a transition in a diluted network (with $K=650$ ) where, because $\bar{g}=-\sqrt{K} g$ [see Eq. (49)], the network lies on a line in the phase diagram. (c) Phase diagram for a threshold-quadratic $(\nu=2)$ network with an excitatory external field $h^{0}=1$. The dashed line shows uniform instability of the fixed point, and the solid line shows the transition to the chaotic phase (shaded area). The dotted (black) line shows the instability of the chaotic phase found by simulations (Gaussian connectivity, $N=6000, h^{0}=1$ ). The inset (d) is the same as (c) for larger values of $|\bar{g}|$. The dashed line shows the existence for a diluted network as in (b). is independent of the magnitude of $h_{0}$ as long as it is positive. In a diluted network, the phase plane is reduced to a single line,

$$
\bar{g}=-g \sqrt{\frac{K}{1-K / N}} .
$$

For a sparse network where $K \ll N, \bar{g}=-\sqrt{K}$ [dashed line in Fig. 2(e)]. Thus, for large values of $K$, as in the balanced network case, the chaotic state at $g>\sqrt{2}$ is always stable.

In Fig. 2(c), the phase diagram for the semiquadratic $(\nu=2)$ is presented for $h^{0}=1$. For low values of $|\bar{g}|$, uniform instability occurs at lower $g$ than chaotic instability, and no stable chaotic phase exists. For larger values of $|\bar{g}|$, a critical transition between a fixed point and chaotic dynamics exists. For larger values of gain $g$, the dynamics always diverges; this instability is shown by numerical simulation in the phase diagram. For a diluted network, where $|\bar{g}|=\mathcal{O}(\sqrt{K})$, there is always a chaotic transition, as shown in the inset of Fig. 2(c).

\section{Analytical evaluation of the Lyapunov exponent}

In the single-population case, the evaluation of the Lyapunov exponent is also relatively simple. In this case, the single-component Hamiltonian is given by $\mathcal{H}=$ $-\left(\partial^{2} / \partial \tau^{2}\right)+W(\tau)$, with a quantum potential $W=$ $-\partial^{2} V / \partial q^{2}$, where $V$ is the classical potential [Fig. 3(a)], which can be easily evaluated once $q(\tau)$ is known [Fig. 3(b)]. Indeed, for the semilinear network, we find that the ground state is negative for $g<\sqrt{2}$ and positive otherwise, implying that $\lambda_{L}$ is negative for $g<\sqrt{2}$ and changes sign to a positive value for $g>\sqrt{2}$, as expected in a chaotic state [see Figs. 2(c)-2(f)]. For the nonlinear case with $\nu=2$, the Lyapunov exponents are calculated near and above the transition in Sec. VII.

\section{E. Numerical simulations}

Numerical integration of the full network of equations in Eq. (32) verifies our theoretical predictions. For a threshold- linear network, when $g<\sqrt{2}$, the network settles into a fixed point, with the expected mean and variance of the local quenched fields [Fig. 4(a)]. When $g>\sqrt{2}$, chaos settles with temporal fluctuations that increase with $g$. The population-averaged currents remain almost constant with small finite-size fluctuations [Figs. 4(b) and 4(c)]. The chaotic behavior is characterized first by the decay of the autocorrelation [Fig. 4(d)], which agrees with the theoretical $q(\tau)$, and second by a positive LE. The latter was calculated from simulations using Wolf's algorithm [36]. The resultant values $\lambda_{L}=0.121$ and 0.225 for $g=2.2$ and 3.0, respectively, agree well with the values 0.126 and 0.232 obtained by numerically calculating the ground state 

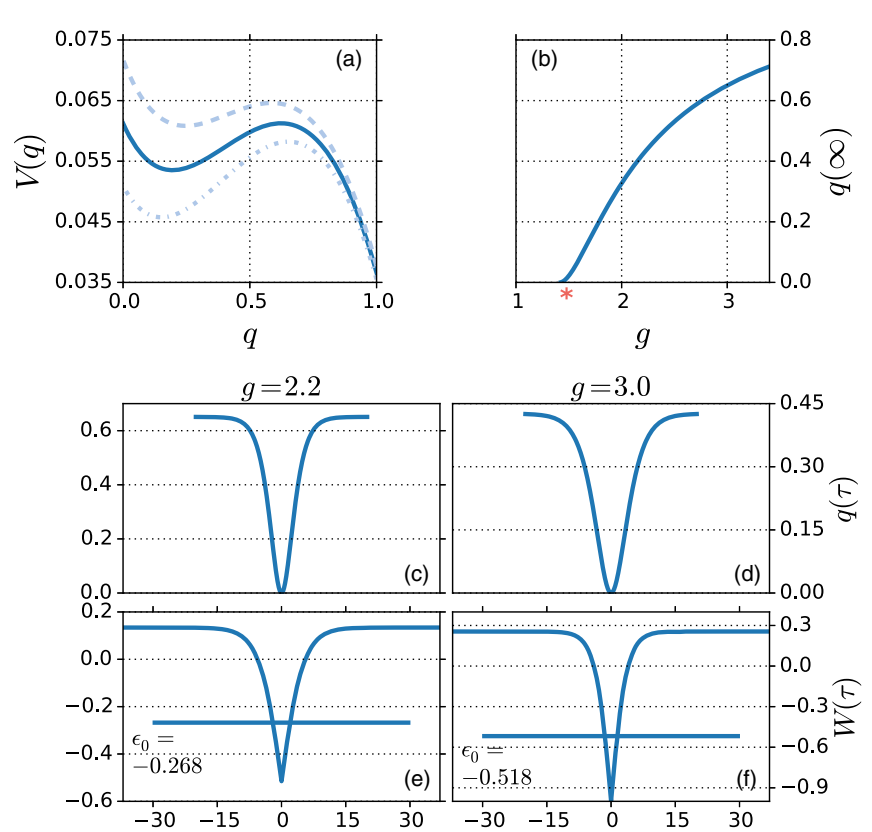

FIG. 3. Calculation of the largest Lyapunov exponent for a threshold-linear network. (a) Numerical integration of Eq. (45). The value of $x$ is determined through the requirement that the maximum of the potential at nonzero $q$ equals its value at zero $q$. Compare the form of the potential with the exact value of $x$ (solid line) with those calculated with $x$ deviating by $\pm 1 \%$ from the correct value (dashed lines). (b) Numerical solution for the normalized variance $q(\infty)$ as a function of $g$. (c)-(f) The normalized autocorrelation function $q(\tau)$ found by integrating the equation of motions (45) using the correct value of $x$ for two values of $g$ (top), and the corresponding quantum potential $W=-\partial^{2} V / \partial q^{2}$ (bottom). The ground-state energies $\epsilon_{0}$ (horizontal lines) were found numerically. In both cases, they are negative, implying a positive Lyapunov exponent.

of the potential $W(\tau)$ [Fig. 2(d)]. Simulation of a semiquadratic network also verifies our analytical results. Simulation results near and above the chaotic transition are given in Sec. VII.

Randomly diluted networks.-The above numerical results were for Gaussian-distributed synaptic connections. However, as shown above, in the limit of large mean number of connections per neuron, $K$, the DMFT is expected to hold also for randomly diluted networks, in which case, $g$ and $\bar{g}$ are related through Eq. (7) (dashed lines in Fig. 2). In the case of a single population with a threshold-linear transfer function, comparing the behaviors of different connectivity schemes (Gaussian, sparse, and dense dilution) is relatively simple since the normalized autocorrelation $q(\tau)$ depends only on $g$, not on $\bar{g}$ (see above). These expectations are borne out by our numerical simulations, shown in Fig. 5. For simulations performed on the network with the same variance in their connectivity, the calculated normalized autocorrelation $q(\tau)$ is identical in the Gaussian network and the randomly diluted networks with both $p=K / N=0.05$ (sparse network) and $p=0.8$ (dense network).
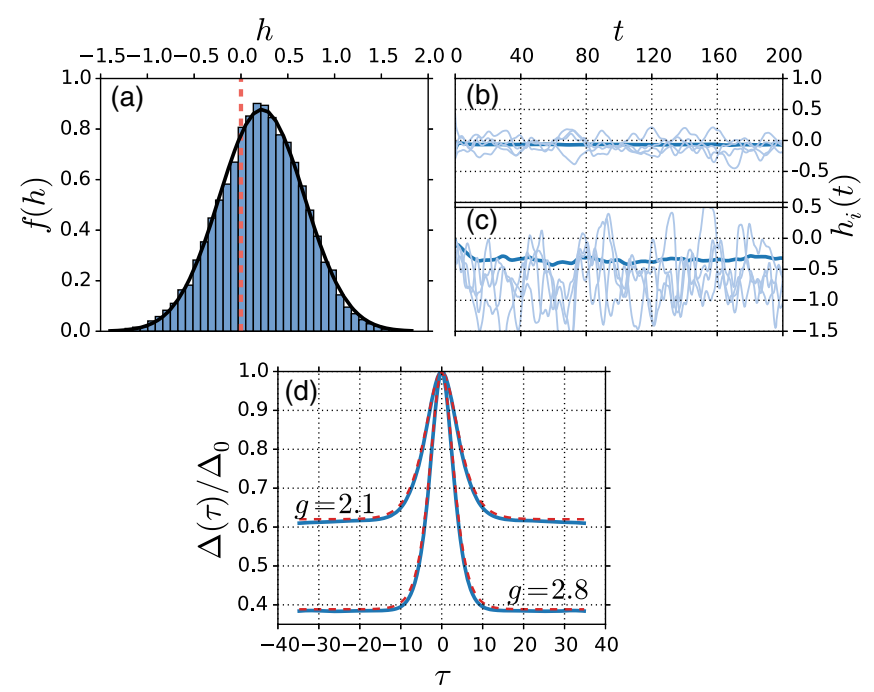

FIG. 4. Numerical simulations of a single population with a linear-threshold transfer function. (a) Distribution of the local fields $h^{i}$ in the fixed-point regime. The thick curve shows normal distribution given the theoretical mean and variance obtained from the mean field theory. The dashed (red) vertical line marks the firing threshold, which is taken to be zero. (b,c) Activities of two networks with gains $g=2.1$ and $g=2.8$, respectively. Bold lines show spatially averaged local fields; thin lines are local fields of a sample of four neurons. (d) The normalized ac function, $\Delta(\tau) / \Delta_{0}=1-q(\tau)$, for two values of gain parameters as in (b) and (c). Solid black lines are the solutions of the equation of DMFT, Eq. (45), superimposed on simulation results (dashed lines). We show simulations performed on a network $(N=6800)$ with Gaussian-distributed connections and $\bar{g}=\sqrt{K} g$, where $K=680$ and the external field $h^{0}=1$.
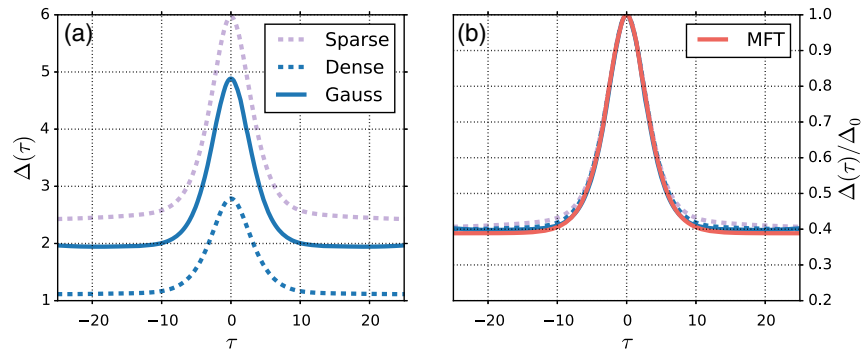

FIG. 5. Robustness to changes in the synaptic distribution. (a) Autocorrelation function for sparse $(p=0.05)$, dense $(p=0.8)$, and Gaussian connectivity networks $(N=7000$, $\left.h^{0}=1\right)$. The gains were chosen such that all networks have the same variance for the connectivity distribution $(g=2.8$ for the Gaussian distribution and $g \sqrt{1-p}=2.8$ for the randomly diluted networks). (b) Normalized autocorrelation function $1-q(\tau)=\Delta(\tau) / \Delta_{0}$ compared with the solution of the DMFT.

\section{TWO POPULATIONS WITH THRESHOLD-LINEAR TRANSFER FUNCTION}

Here, we address briefly the application of the general theory to the case of a two-population network with one 
excitatory (denoted as $E$ ) and one inhibitory $(I)$ population [Fig. 1(c)]. For simplicity, we study the example of the semilinear transfer function. Generalization to other values of $\nu$ is straightforward, as presented in Sec. III.

\section{A. Fixed point and its stability}

The fixed-point equations for the variance and mean of the synaptic current are

$$
\begin{aligned}
& \Delta_{k}=\sum_{l=E, I} g_{k l}^{2} \Delta_{l}\left\langle\left[z+x_{l}\right]_{+}\right\rangle_{z}, \quad k=E, I, \\
& m_{k}=\Delta_{k}^{\nu / 2}\left\langle\left[z+x_{k}\right]_{+}\right\rangle_{z}, \quad k=E, I,
\end{aligned}
$$

along with the definition of $x_{k}$,

$$
x_{k} \sqrt{\Delta_{k}}=\sum_{l} \bar{g}_{k l} m_{l}+W_{k} m_{0} .
$$

The eigenvalues of the stability matrix $M_{k l}=g_{k l}^{2} H\left(-x_{l}\right)$ are given by

$$
\Lambda_{ \pm}=\frac{1}{2}\left[M_{E E}+M_{I I} \pm \sqrt{\left(M_{E E}-M_{I I}\right)^{2}+4 M_{I E} M_{E I}}\right] .
$$

Note that the two eigenvalues are real. For a fixed point to be stable, $\Lambda_{+}<1$. Thus, the transition to chaos occurs at parameters s.t. $\Lambda_{+}=1$. When this eigenvalue becomes larger than 1 , one must solve the DMF equations for the chaotic state.

\section{B. Chaotic state}

The DMF equations can be written as

$$
\begin{gathered}
\left(1-\frac{\partial^{2}}{\partial \tau^{2}}\right) q_{k}(\tau)=1-\tilde{g}_{k E}^{2} C_{E}(\tau)-\tilde{g}_{k I}^{2} C_{I}(\tau), \\
C_{k}(\tau)=\left\langle\left\langle\left[\sqrt{q_{k}(\tau)} y+\sqrt{1-q_{k}(\tau)} z+x_{k}\right]_{+}\right\rangle_{y}^{2}\right\rangle_{z},
\end{gathered}
$$

for $k \in\{E, I\}$, where

$$
q_{k}(\tau)=1-\Delta_{k}(\tau) / \Delta_{k}(0)
$$

are the normalized autocorrelation functions for each population, and here we set

$$
\tilde{g}_{k l} \equiv g_{k l} \sqrt{\frac{\Delta_{l}(0)}{\Delta_{k}(0)}} .
$$

Unlike the simple case of a single population, no classical potential function can be defined for the above two-particle motion in Eq. (54). Nevertheless, the dynamical equations can be integrated numerically, iteratively finding the values for $x_{k}$ that yield the desired asymptotic behavior of the normalized variance,

$$
q_{k}(\infty)=1-\Delta_{k}(\infty) / \Delta_{k}(0) .
$$

Likewise, in general, the Hamiltonian governing the Lyapunov susceptibility $\mathbf{G}(\tau)$ is not Hermitian. However, as stated above, we expect the ground state to be real since the elements of $\mathbf{G}(\tau)$ are non-negative by definition, and a complex ground state would mean oscillations around zero (see Appendix C).

\section{Numerical results}

Below we describe the chaotic state of this network, based on numerical investigations. In these simulations, we focus on the balanced regime, in which all the mean contribution of each population to the local input is of order $\sqrt{K}$. In this case, to leading order, excitation and inhibition cancel each other, and the mean activity of each population is given by

$$
m_{E}=-\left[J^{-1} W\right]_{E} m_{0}
$$

and

$$
m_{I}=-\left[J^{-1} W\right]_{I} m_{0},
$$

where $W=\left[W_{E} W_{I}\right]^{T}$ is the vector of feedforward connections, and $J$ is the $2 \times 2$ matrix of the mean recurrent connections, as defined in the diluted model above. The balance conditions on the parameters are

$$
\begin{gathered}
\operatorname{det} \mathbf{J}<0, \\
J_{E I} W_{I}<J_{I I} W_{E},
\end{gathered}
$$

and

$$
J_{I E} W_{E}<J_{E E} W_{I} .
$$

To demonstrate the properties of the two-population system, we fix all the values of the connections except for a global gain $g$, and $\alpha$, which controls the excitatory connections. For $\alpha=0$, the network is reduced to the single inhibitory network with the parameters used in Sec. V, and for $\alpha>0$, the excitatory connections are $J_{E E}=J_{I E}=$ $W_{E}=\alpha J_{I I}=\alpha g$. In Fig. 6(a), we show a section of the phase space, with the critical $g_{c}(\alpha)$ line. The critical curve is calculated by solving the eigenvalues of the stability matrix (53) for each value of $\alpha$. Figure 6(b) shows an example of the stability for $\alpha=0.55$. Numerical simulations confirm the theoretical results, as seen in Fig. 6(c). For convenience of comparison, parameters were chosen so that they correspond to the same network parameters studied in 

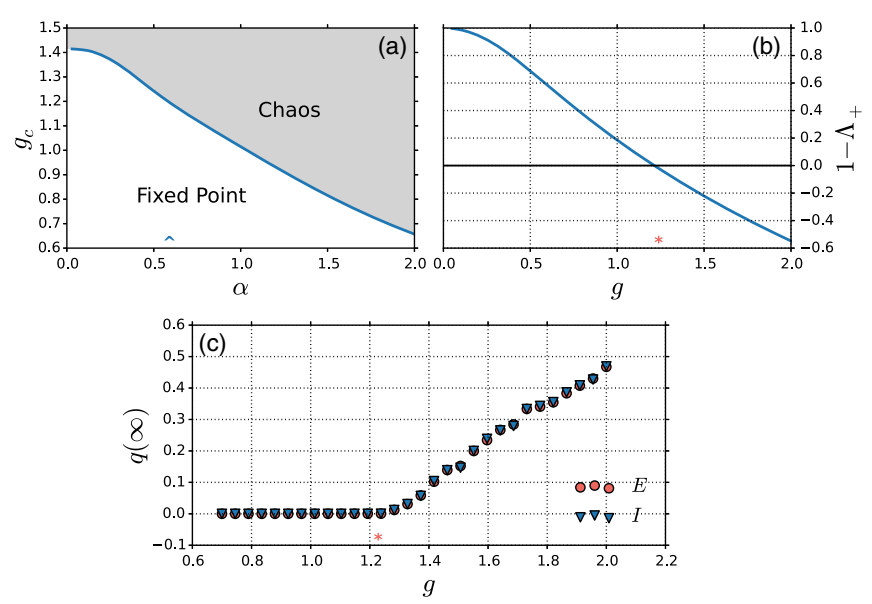

FIG. 6. Transition to chaos in a system of two populations. Parameters used: $\quad J_{E E}=J_{I E}=W_{E}=\alpha g, \quad J_{I I}=-g$, $J_{E I}=-1.11 g$, and $W_{I}=0.44 g$. Note that $g$ is a global gain multiplying all the synapses; $\alpha$ denotes the excitatory efficacy. (a) The critical value of $g$ as a function of the excitatory efficacy $\alpha$. For $\alpha=0$, the network is identical to the single inhibitory network and the phase transition occurs at $g=\sqrt{2}$. (b) Largest eigenvalue of the stability matrix (53) for a network with $\alpha=$ 0.55 [marked by " ") in panel (a)]. MF predicts a phase transition when $\Lambda_{+}=1$ (asterisks). (c) Normalized variance $q_{k}(\infty)$ for inhibitory (blue triangles) and excitatory (red circles) populations as a function of the gain calculated from network simulations with $\alpha=0.55$. It was calculated from simulation of a balanced diluted network with $N_{E}=N_{I}=3500, K=700$, connectivity parameters as in panel (b), and external activity $m_{0}=1$. Theory predicts a phase transition at $g=1.21$ (asterisks), as seen in panel (b).

Refs. $[8,9]$. Unlike the binary network in which no stable fixed point exists for any $g$, in the threshold-linear network, a transition to chaos occurs at $g=1.21$ [Figs. 6(b) and 6(c)]. Figure 6(c) indicates that the normalized variances of the two populations are very similar to each other (see discussion in Sec. 10 below).

Figure 7 shows an example of the chaotic fluctuations in the two-population network. The same parameters were used as in Figs. 6(a) and 6(b) with $g=1.6$, within the chaotic phase. As expected, inputs into neurons from both populations show large fluctuations, while the mean activity of each population is constant up to fluctuations of order $1 / \sqrt{K}$ [Fig. 7(a)]. The autocorrelation of both population decays monotonically with $|\tau|$ to an equilibrium value [Fig. 7(b)]. A signature of a dynamical balanced state is the substantial synchrony in the fluctuations of the excitatory and inhibitory mean activities [Fig. 7(c)]. Consistent with Fig. 6(c) above, Fig. 7(b) shows that the autocorrelation functions of the two populations are nearly proportional to each other, implying that the normalized autocorrelation functions, $q_{E}(\tau)$ and $q_{I}(\tau)$, are approximately equal (as observed recently also by Ref. [37]; see Sec. VII).
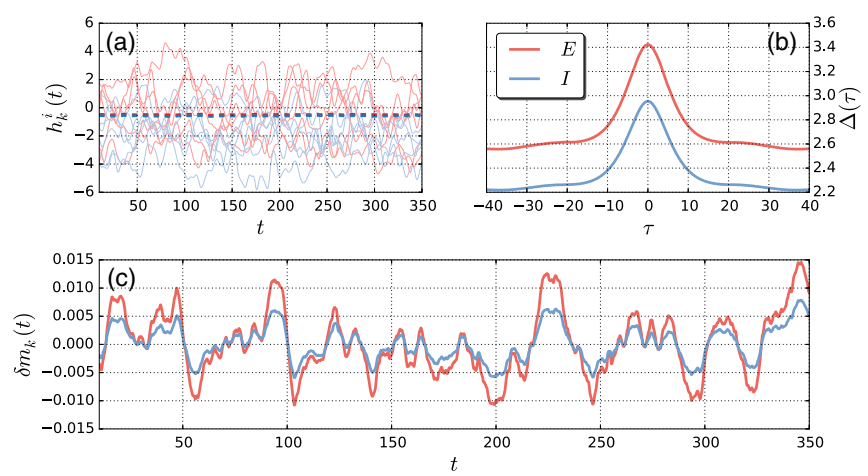

FIG. 7. Fluctuations of an E-I balanced network with linearthreshold transfer function. Same parameter set as in Fig. 6(c). (a) Traces of the local fields of six neurons from excitatory (red) and inhibitory (blue) populations. Dashed lines show mean input into each population. (b) Time-lagged autocorrelation function of two populations computed from the simulation. (c) Trace of the fluctuations in the spatially averaged activities $\delta m_{k}(t) \equiv m_{k}(t)-\left\langle m_{k}(t)\right\rangle_{t}$ of both populations [same color codes as (a)]. Simulations of network are similar to the one used in Fig. 6(b), with $g=1.6$.

\section{CRITICAL BEHAVIOR AT THE ONSET OF CHAOS}

In this section, we analyze the characteristics of the system at the onset of the chaotic state and ask what features determine the critical properties of this transition. As will be seen below, these properties depend on the shape of the single neuron transfer function near the origin. As such, the class of power-law functions defined in Eq. (33) can represent any continuous threshold function.

Absence of stable chaotic phase.-Before we explore the critical properties near the transition to chaos, we note that not all transfer functions allow a stable chaotic solution. For example, Eq. (25) may not have a solution satisfying all boundary conditions. In these instances, the dynamics is either stationary or explosive (i.e., "epileptic"). An example of such behavior is the exponential curve $\phi(x)=e^{x}$, as shown in Appendix D. On the other hand, all $\phi(x)$ that saturate as $x \rightarrow \infty$ are expected to exhibit a stable chaotic state for large values of $g$.

In the following, we focus our study on a transfer function of the type (33), with $\nu>1 / 2$, which exhibits a phase transition from FP to stable chaos.

\section{A. Critical properties: Single population}

When $\nu>1 / 2$, the critical properties near the transition to chaos depend on the value of $\nu$. To see this, we first consider the case of a single inhibitory population. The critical value of $g$ is given by the value at which $g^{2}\left\langle\left[\phi^{\prime}(\sqrt{\Delta} z+u)\right]^{2}\right\rangle=1$ [see Eq. (39)]. As the chaotic state approaches the critical $g$ from above, the amplitude of the time-dependent fluctuations becomes small; hence, we can expand the MF equations in powers of $q(\tau)$ (which is 
small at all $\tau$ ). In addition, we expand the equations in the small static parameter $\delta x \equiv x-x_{c}$ and the bifurcation parameter $\epsilon \equiv g^{2} / g_{c}^{2}-1$.

To leading order, the DMF equation (54) takes the form (see Appendix E)

$$
\frac{\partial^{2} q(\tau)}{\partial \tau^{2}}=a(\epsilon)+b \epsilon q(\tau)+c q^{\rho}(\tau)
$$

where $b$ and $c$ are parameters of order unity, and the exponent $\rho$ obtained from expansion of the firing-rate autocorrelation, $C(\tau)$ (see Appendix E), is

$$
\rho= \begin{cases}\frac{3}{2} & \frac{1}{2}<\nu \leq \frac{3}{2} \\ 2 & \frac{3}{2}<\nu .\end{cases}
$$

The constant term $a$ vanishes at the transition and depends on $\epsilon$. In order for a nontrivial solution to exist, all the terms in Eq. (60) should be of the same order of $\epsilon$; hence, the time scale of $q(\tau)$ should scale as $\tau_{\text {eff }} \sim 1 / \sqrt{\epsilon}$. The amplitude of $q(\tau)$ should scale as $\epsilon^{2}$ for $\nu \leq 3 / 2$ and as $\epsilon$ for larger $\nu$. Finally, $a$ should scale as $O(\epsilon q)$ (as indeed found by an explicit evaluation of $a$; see Appendix E), yielding the following scaling behavior:

$$
q(\tau)=\epsilon^{1 /(\rho-1)} f\left(\frac{\tau}{\sqrt{\epsilon}}\right),
$$

where $f(x)$ is of order 1. The Hamiltonian in Eq. (29) scales as

$$
W(\tau)=\epsilon F\left(\frac{\tau}{\sqrt{\epsilon}}\right)
$$

where $F(x)$ is some other function of order 1. Equation (63) implies that the largest Lyapunov exponent, Eq. (31), scales as

$$
\lambda_{L}=O(\epsilon)
$$

We have confirmed the above predictions numerically, for the cases of $\nu=1$ (Fig. 8) and $\nu=2$ (Fig. 9).

In this work, we assume a synaptic transfer function of the form (33). In Ref. [6], the variance of a network with a sigmoid synaptic transfer function, $\phi(h)=\tanh (h)$, and $h^{0}=0$, was shown to scale as $\Delta(\tau)=\epsilon f(\tau / \sqrt{\epsilon})$ and its LE as $\lambda_{L}=O\left(\epsilon^{2}\right)$. This behavior results from the $h \rightarrow-h$ symmetry of the system, which implies that $\Delta_{0}$ vanishes at the transition. This symmetry is not expected to hold in biological networks. Thus, the scaling shown in Eqs. (62) and (64), in which the LE is larger than in the symmetric case, reflects the behavior in generic neuronal networks.

In Ref. [38], the authors study the distribution of equilibria points above criticality, for a single population with a sigmoidal transfer function. An interesting result is
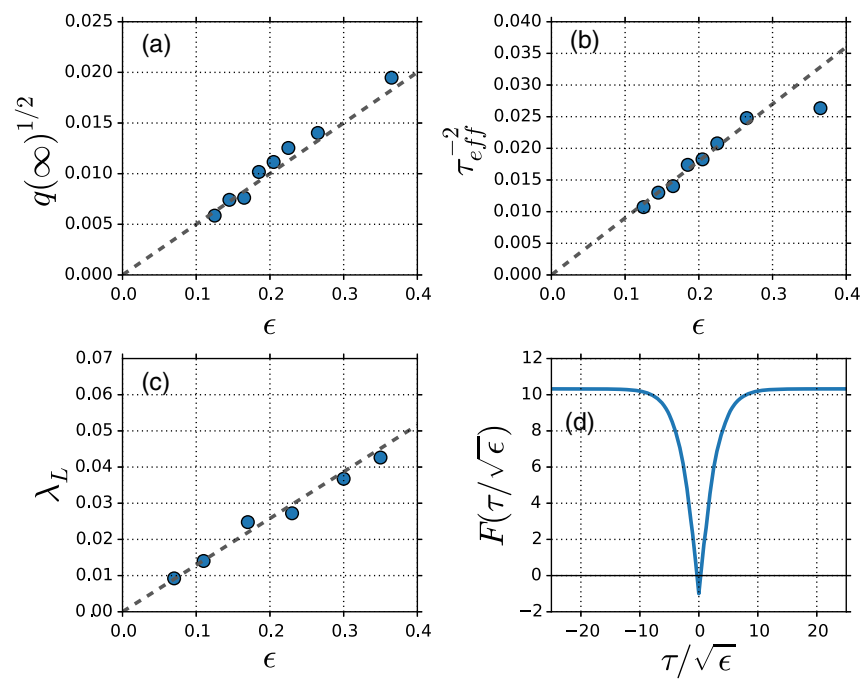

FIG. 8. Critical behavior of a single inhibitory population with linear transfer function. Normalized variance (a) network relaxation time (b), and largest Lyapunov exponent (c) as a function of the distance from the critical point $\epsilon=\frac{1}{2} g^{2}-1$. Circles show the average over 20 simulations (Gaussian connectivity, $N=6000$, $h^{0}=1$ ), and dashed lines show theoretical predictions (see Appendix E). (d) Rescaled one-dimensional quantum potential $F(\tau / \sqrt{\epsilon})$, Eq. (63), for the Hamiltonian in Eq. (29).

that the mean (with respect to realizations of $J_{i j}$ ) number of equilibria behaves just like the Lyapunov exponent. Consequently, Eq. (64) may also elucidate the topological complexity of the flow above criticality for threshold power-law transfer functions.

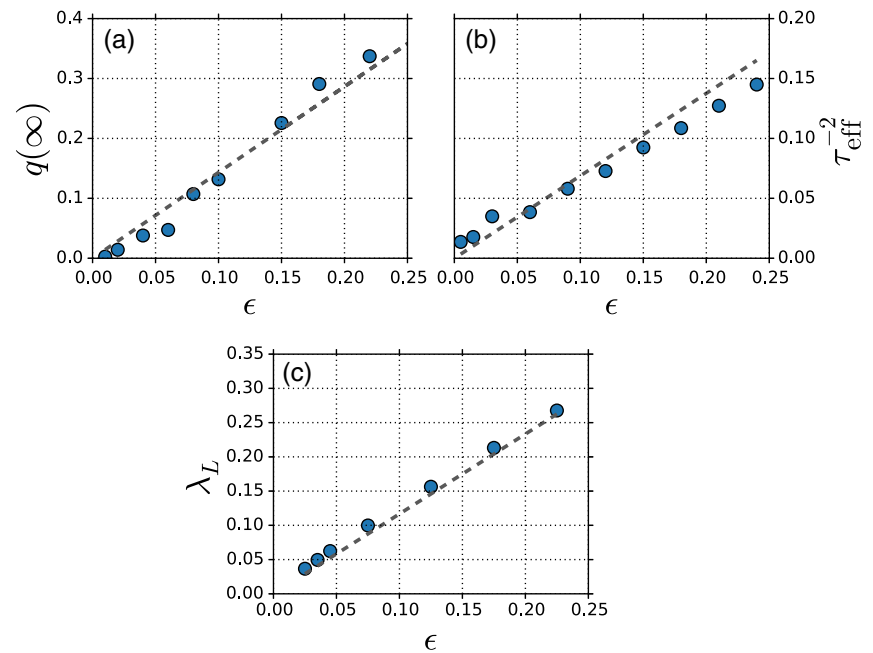

FIG. 9. Critical behavior of a single inhibitory population with quadratic $(\nu=2)$ transfer function. Normalized variance (a), network relaxation time (b), and LE (c) for small $\epsilon \equiv g^{2} / g_{c}^{2}-1$ gain above the critical point. Circles show the average over 20 simulations (Gaussian connectivity in the balance regime, $\left.N=6000, K=1200, h^{0}=1\right)$, and dashed lines show theoretical predictions (see Appendix E). 


\section{B. Multiple populations}

The above critical properties were derived for the case of a single population. However, we argue that these properties hold also for multiple populations. In the case of several populations, transition to chaos occurs when the largest eigenvalue of the stability matrix, $\mathbf{M}$ [Eq. (22)], equals 1 (and assuming the uniform stability still holds). Near the transition (on the chaotic side), this eigenvalue is slightly larger than 1, while the real part remains stable. To leading order, the unstable direction, $R^{1}$, in the space of $q_{k}(\tau)=$ $1-\Delta_{k}(\tau) / \Delta_{k}(0)$ remains the same as the marginally stable eigenvector at the critical point. Hence, near the transition, the dominant direction of temporal fluctuations is along the critical eigenvector, and the dynamic equations collapse into one dimension, similar to the one population case. In general, we expect that all $q_{k}$ have nonzero components on the critical direction; hence, $q_{k}(\tau) \approx q(\tau) R^{1}$ and the critical properties are the same as the single-population properties above (see Appendix E). Similar arguments apply to the scaling properties of the quantum Hamiltonian; hence, the LE scales as Eq. (64). These results are supported by simulations of an excitatory-inhibitory network, defined in Sec. VI. The simulations displayed in Fig. 10 demonstrate the universality of the critical properties of the transition to chaos. In the specific case of a threshold-linear model, we

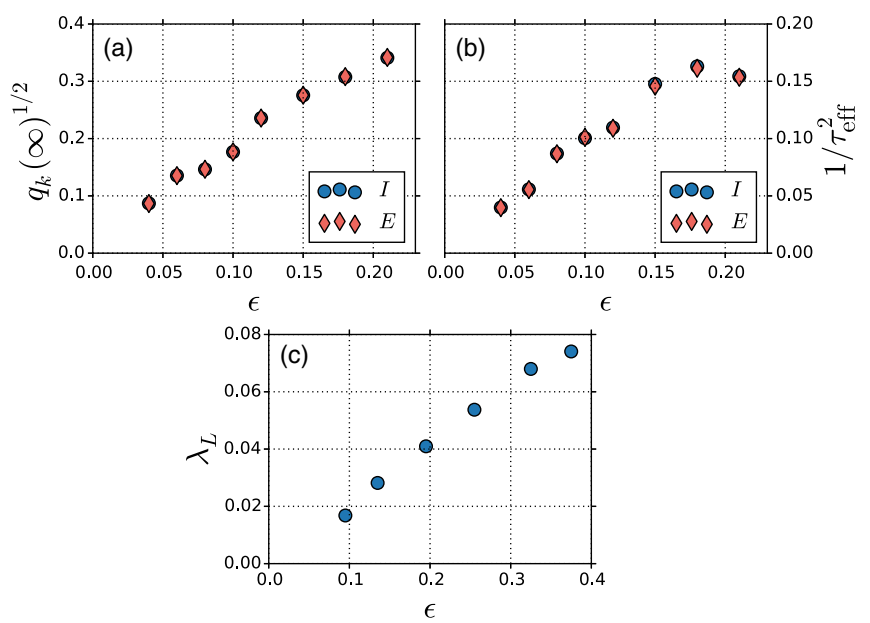

FIG. 10. Critical behavior of a network with two populations and a linear transfer function. Diamond (red) and circles (blue) denote excitatory and inhibitory populations, respectively. (a) The normalized variances of the two populations, $q_{E}(\infty)$ and $q_{I}(\infty)$, show the same critical behavior (a linear rise from zero) as the single population [Eq. (62)]. Because of the linearity, the normalized autocorrelations are nearly equal to each other near the critical point (see Appendix E). (b) Network relaxation times of both populations are similar at the scaling limit. (c) LE of the entire network calculated over all populations, Eq. (27), scale as a single population, Eq. (64). We show simulations conducted on a network with a diluted connectivity matrix $(N=3600$, $K=600$ for each population) and a parameter set as in Figs. 7 and 6(c). find for a wide range of parameters, $R^{1} \approx(1,1,1, \ldots, 1)$, implying that near the transition, the normalized autocorrelations of all populations are not only proportional but are expected to be approximately equal to each other, as detailed in Appendix E.

Note that the one-dimensional character of the chaotic fluctuations is exact only asymptotically close to the transition. Away from the transition, the nonlinearity of $C(q)$ couples the unstable mode to the other modes, inducing fluctuations in all modes. Interestingly, we find numerically that in many cases, even far from the transition, the autocorrelations are still close to being equal, $q_{k}(\tau) \approx q(\tau)$, as is apparent in the example of Figs. 6(c) and 7(b). Similar observations have been made recently in Ref. [37].

\section{TRANSITION TO CHAOS IN SPIKING NETWORKS}

In this section, we ask under what conditions the transition to chaos observed in the rate dynamics occurs also in spiking networks. In contrast to rate models, the membrane potentials of spiking networks are not at a fixed point as long as some of the neurons are active. Hence, in general, there is no transition from fixed point to chaos. An exception is the case of networks with slow synapses. If the synaptic time constant is long compared to the spiking dynamics, it is possible that for low synaptic strength, the spiking dynamics is averaged out at the level of the slow synaptic currents, which will therefore stay approximately constant. Thus, there might be a critical $g$ in which the state with almost constant synaptic currents undergoes an instability leading to a chaotic (or at least temporally irregular) state in which temporal fluctuations of the synaptic currents are large even at the scale of the synaptic time constant.

To explore the possibility of transition to chaos in spiking networks with a slow synaptic time constant, we consider a network of spiking neurons that fire with inhomogeneous Poisson statistics, specified by the instantaneous rate of each neuron, $r^{i}(t)=\phi\left(h^{i}(t)\right)$. We assume that the network is in an asynchronous state [39] and that the typical firing rate is large compared with the inverse synaptic time constant, $\tau_{s}^{-1}$.

Focusing on single inhibitory populations, we write the dynamic equation of the local fields as

$\tau_{s} \frac{d}{d t} h^{i}(t)=-h^{i}(t)+\sum_{j=1}^{N} \mathcal{J}^{i j} \xi^{j}(t)+\tau_{0} \bar{g} r(t)+h^{0}$,

where $\mathcal{J}^{i j}$ is either a Gaussian-distributed connection or the corresponding randomly dilute network, as appears in Eq. (32) above. The spike train $\xi^{j}(t)=\sum_{k} \delta\left(t-t_{k}^{j}\right)$ of the presynaptic neuron $j$ at times $t_{k}^{i}$ represents the random Poisson process of neuron $j$. The nonlinear rate function is 
given by the rectified linear transfer function $\phi(x)=$ $\tau_{0}^{-1}[x]_{+}$, where $\tau_{0}$ is a microscopic time constant, which is related to the inverse slope of the single-neuron $f-I$ curve. Note that in this model, the connections have units of time; hence, we define $g^{2}=N\left\langle\left(\mathcal{J}^{i j}\right)^{2}\right\rangle \tau_{0}^{-2}$ so that $g$ (and $\bar{g}$ ) is dimensionless. For simplicity of notation, in the following, we measure rates and times in units of $\tau_{0}$; i.e., we use units such that $\tau_{0}=1$.

To understand the effect of spiking dynamics, we can separate the synaptic input into a rate contribution and a spiking noise contribution,

$$
\eta_{i}(t)=\eta_{i}^{r}(t)+\eta_{i}^{s p}(t)
$$

where

$$
\begin{aligned}
\eta_{i}^{r}(t) & =\sum_{j=1}^{N} \mathcal{J}^{i j} \phi\left(h_{j}(t)\right), \\
\eta_{i}^{s p}(t) & =\sum_{j=1}^{N} \mathcal{J}^{i j}\left(\xi^{j}(t)-\phi\left(h_{j}(t)\right)\right) .
\end{aligned}
$$

The autocovariances of the two terms on the right-hand side of Eq. (66) are

$$
\left\langle\eta_{i}^{r}(t) \eta_{i}^{r}(t+\tau)\right\rangle=g^{2} C(\tau)
$$

and

$$
\left\langle\eta_{i}^{s p}(t) \eta_{i}^{s p}(t+\tau)\right\rangle=g^{2} r \delta(\tau),
$$

where $C(\tau)=\left\langle\phi_{i}(t) \phi_{i}(t+\tau)\right\rangle$ is the autocorrelation of the rate functions given by Eq. (26) (in units of $\tau_{0}^{-2}$ ), and $\delta(\tau)$ is the Dirac delta function. The last equation results from the average over the Poisson process, $\left\langle\left(\xi^{j}(t)-\phi\left(h_{j}(t)\right)\left(\xi^{j}(t+\tau)-\phi\left(h_{j}(t+\tau)\right)\right\rangle=\phi\left(h_{j}(t)\right) \delta(\tau)\right.\right.$. Thus, the Poisson noise is equivalent to an additive white noise with amplitude $g^{2} r$, where $r$ is the population averaged rate $r$ (in units of $\tau_{0}^{-1}$ ). Thus, the spiking noise can be incorporated into the dynamic mean field theory, yielding

$$
\left(1-\tau_{s}^{2} \frac{\partial^{2}}{\partial \tau^{2}}\right) \Delta(\tau)=g^{2} C(\tau)+g^{2} r \delta(\tau)
$$

\section{A. Perturbation analysis of the spiking noise}

As explained above, we are interested in the regime of large $\tau_{s}$ (which is the synaptic time constant relative to $\tau_{0}$ ). This limit can be illuminated by writing the rescaled mean field dynamics,

$$
\left(1-\frac{\partial^{2}}{\partial t^{2}}\right) \Delta(\tau)=g^{2} C(t)+\frac{g^{2} r}{\tau_{s}} \delta(t)
$$

where we have scaled time so that $t=\tau / \tau_{s}$. The above equation demonstrates that the contribution of the Poisson noise (proportional to $g^{2} r / \tau_{s}$ ) is small relative to the rate contributions (which are proportional to $g^{2} r^{2}$ ), and the ratio between the two is of the order of $\left(r \tau_{s}\right)^{-1}$.

For gain values above $g_{c}=\sqrt{2}$, and $r \tau_{s} \gg 1$, the noise contribution will be small compared to the unperturbed rate autocorrelation given by the solution of Eq. (45) [Fig. 11(a)]. Below $g_{c}$, the only time dependence comes from the spikes. To study this regime, we consider the spikes contribution as a small perturbation around the
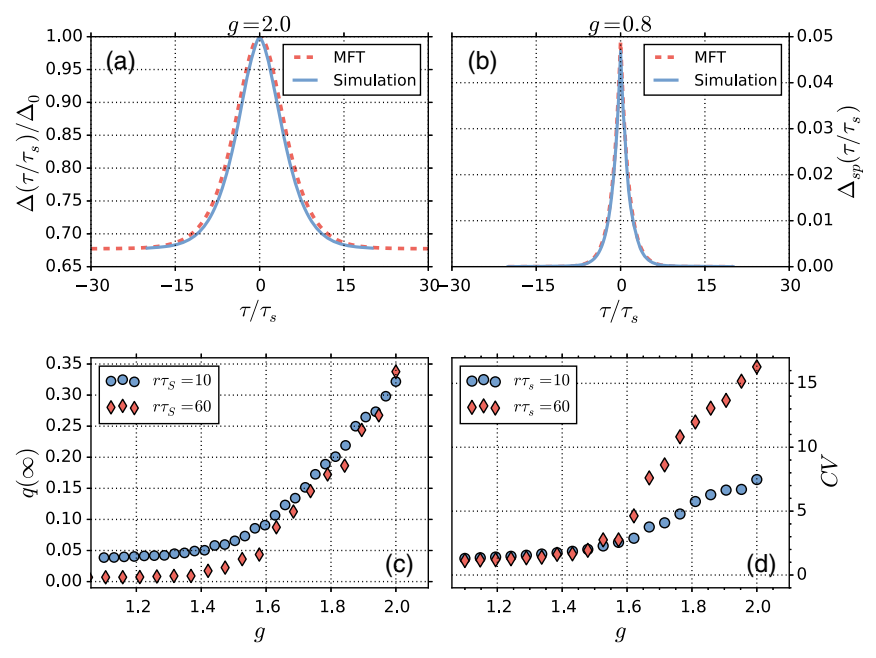

FIG. 11. Network of spiking neurons with inhomogeneous Poisson statistics. Simulation results for a network that follows the dynamic equation in Eq. (65). (a) Above the critical point of the underlying rate dynamics, $g_{c}=\sqrt{2}$, the rate term dominates the DMF equation, Eq. (72), and the autocorrelation calculated from simulation $\left(r \tau_{s}=30\right.$, solid line) is fully explained by autocorrelations of the rates, found by Eq. (45) (dashed line). (b) For values of $g$ well below the rate transition, the rates do not have intrinsic fluctuations, and the fluctuations in the fields are solely due to the Poisson firing. MFT results of Eq. (74) (dashed line) agree with simulations $\left(r \tau_{s}=30\right.$, solid line). In intermediate values of $g$, rate and spike fluctuations interact nonlinearly. (c) Normalized variance of the local fields $q(\infty)$ from simulations of networks at different gain levels in the transition region. For higher levels of $r \tau_{s}$, the crossover between variance dominated by the Poisson process and variance dominated by the fields dynamics becomes sharper. (d) The coefficient of variation $(C V)$, Eq. (76), for different values of gain. For low values of $g$, the $C V$ approaches 1 , indicating a Poisson process with (almost) constant rates. For higher values of the gain, the $C V$ is larger, as expected from an inhomogeneous Poisson process. The crossover between the two regimes becomes sharper as $r \tau_{s}$ increases. Simulations are performed using an inhibitory population with Gaussian-distributed connectivity $(N=3500$, $K=700)$ and $\bar{g}=h^{0}=1$. 
static autocorrelation, $\Delta(t)=\Delta_{s t}+\Delta_{s p}(t)$, where $\Delta_{s t}$ is the static solution for $\Delta$ in the rate model, and we expand the equation above to linear order in $\Delta_{s p}(t)$, which yields

$$
\left(1-\frac{\partial^{2}}{\partial t^{2}}\right) \Delta_{s p}(t)=g^{2} C^{\prime} \Delta_{s p}(t)+\frac{g^{2} r}{\tau_{s}} \delta(t)
$$

where $C^{\prime}=H(-x)$ [see Eq. (40)]. Solving Eq. (73) yields

$$
\Delta_{s p}(\tau)=\frac{g^{2} r}{2 \lambda \tau_{s}} e^{-\lambda\left(|\tau| / \tau_{s}\right)},
$$

where, following Eq. (40),

$$
\lambda^{2} \equiv 1-g^{2} H(-x)
$$

Here, $x$ is the unperturbed net input, Eq. (38), and we have substituted back $\tau=t \tau_{S}$. [Note that $g$ is in units of $\tau_{0}$ ]. Figure 11(b) displays the simulation results for the autocorrelation $\delta \Delta(\tau)=\Delta(t)-\Delta(\infty)$ for $g$ below the critical value, and the theoretical estimates $\Delta_{s p}(\tau)$.

\section{B. Scaling analysis}

Approaching the transition point from below, the Poisson contribution, Eq. (74), is amplified by the divergent response of the fields' dynamics as $\lambda \rightarrow 0$. For a finite $r \tau_{s}$, however, the autocorrelation remains finite at all $g$ and the transition is smoothed. Indeed, Fig. 11(c) shows that $q(\infty)$ increases smoothly as a function of $g$, but its increase becomes sharper the larger $r \tau_{s}$ is. Figure 11(d) shows a similar behavior for a measure of the fluctuations in the spike times, known as coefficient of variation $(C V)$, defined as the ratio between the standard deviation of the ISI distribution, $\sigma_{\mathrm{ISI}}$, and its mean, $\mu_{\mathrm{ISI}}[40]$,

$$
C V=\frac{\sigma_{\mathrm{ISI}}}{\mu_{\mathrm{ISI}}} .
$$

The $C V$ increases from $C V \approx 1$, at small $g$, which is the value for a homogeneous Poisson process, to substantially larger values above $g=\sqrt{2}$, because of the fluctuations in the underlying rates. This increase is smooth but becomes sharper for large values of $r \tau_{s}$.

To study the effect of the small spiking noise on the transition, one needs to perform a nonlinear analysis. Here, we use a scaling analysis, similar to that of a second-order phase transition in equilibrium statistical mechanics. The scaling regime is characterized by two variables, $r \tau_{s} \gg 1$ and $\epsilon \equiv g^{2} / g_{c}^{2}-1,|\epsilon| \ll 1$. We write the normalized variance near the transition as

$$
q(\infty)=\frac{1}{\left(r \tau_{s}\right)^{\alpha}} f(z)
$$

where the scaling variable $z$ is given by

$$
z=r \tau_{s}|\epsilon|^{\beta} \operatorname{sign}(\epsilon)
$$

Far below the transition, $z \rightarrow-\infty$, and according to Eq. (74), $q^{-}(\infty) \sim\left[1 /\left(r \tau_{s}\right) \lambda\right] \sim\left[1 /\left(r \tau_{s}\right) \epsilon^{1 / 2}\right]$, requiring $f(z \rightarrow-\infty) \propto z^{\alpha-1}$ and $\beta(1-\alpha)=\frac{1}{2}$. Similarly, above the transition $z \rightarrow \infty$, and from Eq. (62), $q^{+}(\infty) \sim \epsilon^{2}$, entailing $f(z \rightarrow \infty) \propto z^{\alpha}$ and $\alpha \beta=2$. It follows that the critical exponents are

$$
\alpha=\frac{4}{5}, \quad \beta=\frac{5}{2} .
$$

The behavior of the effective relaxation time of the network can also be treated in a similar manner. In the absence of the spiking noise, the effective time constant of the autocorrelation diverges as the transition is approached from above, as $\tau_{\text {eff }} \sim \epsilon^{-1 / 2}$ [Eq. (62) and Fig. 8(b)]. In the presence of small spiking noise, this time constant never diverges, and we write

$$
\tau_{\mathrm{eff}}^{-1}=\frac{1}{\left(r \tau_{s}\right)^{\gamma}} F(z),
$$

where $\tau_{\text {eff }}$ is the effective correlation time in units of $\tau_{s}$. Here, the critical behavior both below and above transition, $z \rightarrow \pm \infty$, is similar [as can be seen from Eq. (74)], and $\tau_{\text {eff }}^{-1} \sim \lambda \sim \epsilon^{1 / 2}$, implying $F(z \rightarrow \pm \infty) \propto|z|^{\gamma}$ and $\beta \gamma=\frac{1}{2}$, or

$$
\gamma=\frac{1}{5}
$$

Note that the above results predict that at the (rate dynamic) transition $(\epsilon=0)$, the amplitude of the variance vanishes as $\left(r \tau_{s}\right)^{-4 / 5}$ and the effective time constant diverges $\left(r \tau_{s}\right)^{1 / 5}$, respectively. Simulation results that support these analytical predictions are presented in Fig. 12.

\section{DISCUSSION}

Universality of the transition to chaos across network architectures.-The results presented here show the universality of the dynamical transition from a fixed point to chaos in large networks across different network architectures. The theory, as well as simulations, show no dependence on the detailed distribution of the synaptic connectivity beyond the first two moments. In particular, a Gaussian network behaves similarly to randomly dilute networks with either sparse or dense connectivity. The two architectures differ in the mechanism by which fluctuations are not suppressed by the high connectivity. In the Gaussian networks, the mean of the connection distribution is assumed to be smaller by a factor of $1 / \sqrt{N}$ than their variance. In contrast, in the dilute networks, the mean inputs from each population are strong compared to the fluctuations, and the fluctuations are nevertheless potent 

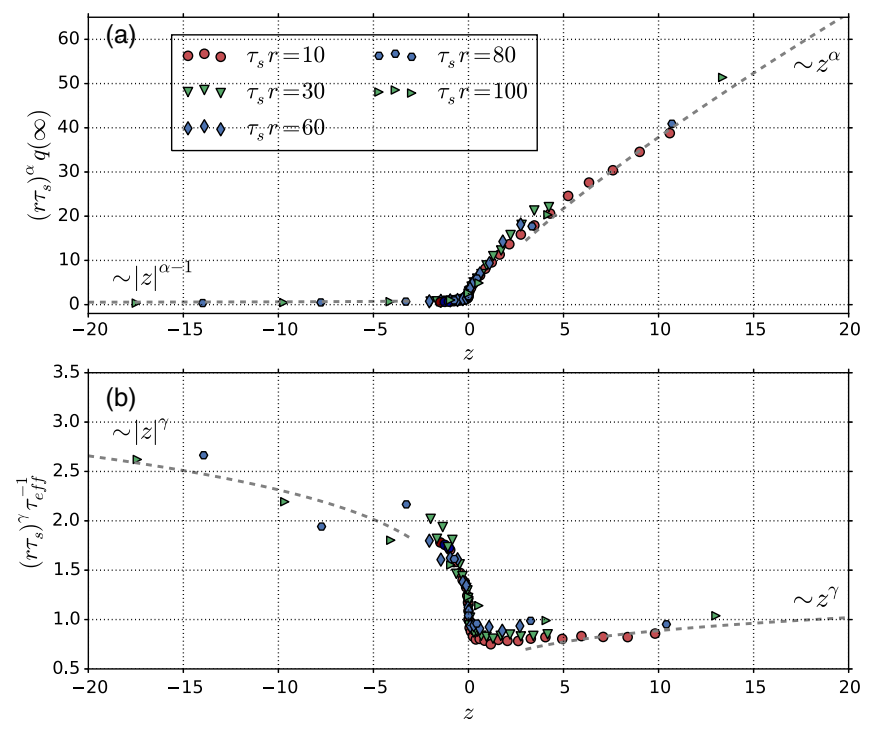

FIG. 12. Scaling behavior near criticality. (a) Rescaled form for the normalized variance, $\left(r \tau_{s}\right)^{\alpha} q(\infty)$ and (b) inverse effective relaxation time $\left(r \tau_{s}\right)^{\gamma} \tau_{\text {eff }}^{-1}$, using the scaling variable $z=\tau_{s} r|\epsilon|^{\beta} \operatorname{sign}(\epsilon)$. The data reveal the underlying scaling functions $f(z)$ and $F(z)$, respectively, consistent with Eqs. (77) and (80). Dashed lines show the predicted asymptotic behavior (see text). Simulations are performed on a Gaussian network of various sizes $\left(N=2000,3500\right.$, and 6000, $\left.h^{0}=1\right)$ and with various synaptic time scales (see legend). Effective relaxation was calculated from simulations by taking $\tau_{\text {eff }}=$ $\int \tau \delta \Delta(\tau) d \tau / \int \delta \Delta(\tau) d \tau$ for $\tau \geq 0$.

because of the dynamic balance of excitation and inhibition. This balancing amplifies not only the temporal fluctuations but also the time-averaged ones. For instance, the neurons in the dilute networks exhibit a broad distribution of time-averaged firing rates. In the threshold linear case, the distribution of the firing rates will have a truncated Gaussian shape with a width that is related to $\Delta_{k}(\infty)$. This balance mechanism is similar to the previously shown balanced state in spiking or binary networks. Here, we show that this process is quite general and holds also in smooth dynamics with graded nonlinearity. It should also be noted that most of the previous work on balanced states assumed sparse connectivity, i.e., $K \ll N$. In this limit, neurons share only a few common sources; hence, the network state is naturally asynchronous. Interestingly, we show here that even dense networks (where, e.g., $K / N$ is as large as 0.8; see Fig. 5) exhibit the same transition from a fixed point to asynchronous chaos, as in sparse or Gaussian networks (see also Refs. $[9,26]$ ). It would be interesting to prove analytically the absence of stable synchronous states in these systems.

The analytical and numerical investigations indicate that the transitions to chaos in a single-population and a twopopulation (E-I) network is of the same nature, and the DMFT predicts that this is true also for networks with a general multiple-population architecture. The extension of the DMFT to multiple populations is similar in spirit to previous work on stochastic multipopulation networks with weak connections (of order $1 / K$ ) [41] and remains valid as long as the number of populations is small compared to $K$. The structure of the DMFT in the case of multipopulation networks is different than in the single-population case in that the dynamics of the autocorrelation function is not governed by a potential; thus, numerically solving these equations is more challenging. Furthermore, although near the transition the autocorrelations behave qualitatively similarly to the single-population case, deep in the chaotic phase, depending on the parameter value, they may not be monotonically decreasing with time but might exhibit damped oscillations. Likewise, in multiple-population networks, the "quantum" operator for the Lyapunov exponent is non-Hermitian, the implications of which needs to be further explored.

Stability against uniform perturbation.-We have focused primarily on asynchronous dynamical states and instabilities driven by the random components of the interactions. However, in the general architectures considered here, the fixed-point state may first undergo an instability associated with the population-averaged perturbations. Interestingly, because of the inherent nonlinearity of our system, the uniform and local degrees of freedom are coupled. As a result, the response to uniform perturbations involves equations that couple the susceptibility of the population mean activities and that of the population activity variances, Eq. (18). This, together with Eq. (22), constitutes the stability conditions for the fixed points of our general architecture. These results can also be interpreted as results regarding the eigenvalue spectrum of the random matrix of effective couplings that incorporate the local gains $\phi^{\prime}\left(h_{l}^{i}\right)$ from linearizing around the fixed points, which are correlated with the random coupling (see also Ref. [42]).

In the examples studied here, the uniform instability leads to a runaway of the dynamics. In general, in the multipopulation case, the fixed-point instability to uniform perturbations may exhibit an instability leading to coherent oscillatory states, and these states may further become destabilized by the random connectivity yielding possibly chaotic states in the form of partially synchronous oscillations. The exploration of these states is beyond the scope of this paper. Finally, we emphasize that in the case of dilute networks, the primary focus of our work, the underlying strong inhibition moves the system away from these instabilities as long as they are in states with excitation-inhibition balance. In particular, multiply stable states occur only under special conditions $[29,43]$.

Spatially modulated networks.-In the multipopulation model, the functional structure of the entire network is given in terms of pairwise connectivity, which depends on the subpopulation membership of the pair. In the brain, connectivity probability also depends on the distance 
between neurons. This distance can be either with respect to physical location or functional, namely, the preferred stimulus of a neuron. Likewise, the external input to neurons is not, in general, homogeneous but may depend on the physical or functional coordinate of each neuron. Because these location dependencies are broad and smooth, the system can be well described by dividing it into columns, each representing many neurons having similar locations. The total system, a hypercolumn, can thus be considered a special case of our general multipopulation architecture. In the large $N$ limit, the sums over columns will turn into smooth integrals over the spatial coordinates. In general, the architecture of a neural circuit, if large enough, will consist of both genuinely discrete subpopulations (e.g., excitatory and inhibitory) and continuously varying coordinates. An example is a model of an orientation hypercolumn in V1 cortex, where the connectivity between neurons depends on the difference in their preferred orientation, and the external input depends on the difference between the preferred orientation and the stimulus orientation [29]. In this case, the balance equations (15) determine the tuning properties of the mean firing rates of the network. The DMFT describes the statistical properties of the spatiotemporal fluctuations in the network dynamics.

The shape of the nonlinear transfer function.-We have found that the existence of a transition from a fixed point to chaos, as well as its critical properties when it exists, depends on the shape of the input-output transfer function near the origin (i.e., near the firing threshold). An important result is that for a transfer function rising as a square root or sharper, i.e., for $\phi(x) \propto x^{\nu}$ with $\nu \leq 1 / 2$, there is no stable fixed point, and the system is also chaotic for small $g$. This raises the interesting question about the value of $\nu$ in biologically relevant networks. In biological applications of rate models, linear, quadratic, or values of $\nu$ larger than 1 have often been used [44-48]. The transfer function $\phi$ is often interpreted as reflecting the $f-I$ curve of a spiking neuron. The linear leaky integrate and fire (LLIF) model $[49,50]$ exhibits a sharp $[1 /|\log (x)|]$ rise from zero, corresponding to $\nu=0$. Our theory predicts that random networks with such $f-I$ curves exhibit chaotic dynamics also for low $g$. In conductance-based (Hodgkin-Huxleytype, or HH-type) models, the behavior of the $f-I$ curve near threshold depends on the type and parameter values of the various nonlinear conductances contributing to the spike generation. In HH-type-I models, often used for modeling cortical neurons, the generic behavior of the $f-I$ curve near threshold is a square root [51], making them borderline cases for a transition at finite $g$. Similar $\nu=1 / 2$ behavior is exhibited also by nonlinear (NLLIF) models [50]. The presence of an adaptation current with a long adaptation time constant results in a linearization of the $f-I$ curve [52,53] and thus corresponds to $\nu=1$. Therefore, slow adaptation currents in randomly connected networks are predicted to stabilize fixed-point states at low $g$. It is interesting to note that the fixed-point equations for the population-averaged activities (14) are stable to linear perturbations even for $\nu<\frac{1}{2}$ because of the smearing of the singularity near threshold by the Gaussian fluctuations. On the other hand, as we have shown here, this smearing is not strong enough to avoid instability of the fixed point of the local activities and fields.

Finally, our prediction that for $\nu \leq 1 / 2$ no stable fixed point exists was derived in the limit of large $K$, where the distribution of local inputs is Gaussian and hence unbounded. However, in a finite system, where $K$ is finite, there will be a finite gap between zero and the smallest input (in absolute value). Hence, in a finite system, there should be a stable fixed point for sufficiently low values of $g$, even for low values of $\nu$. Studying this finite size effect is an interesting topic for future research.

Although the properties of the transition to chaos are determined by the behavior of $\phi(x)$ near threshold, the overall shape of $\phi$ may also affect the system's behavior. For instance, in the threshold linear case, the effect of changing the magnitude of the external input is marginal, as it can be scaled out from the equations determining the chaotic behavior because of the inherent linearity above threshold [see Eq. (39)]. In contrast, when $\phi(x)$ saturates to a finite value at large $x$, large external input pushes neurons to saturation and suppresses the onset of chaos [54]. Also, the unboundedness of the threshold-linear $\phi$ leads to a divergent dynamics for sufficiently large $g$ (see Fig. 2). Furthermore, when $\phi(x)$ grows exponentially with $x$, this instability sets in as soon as the fixed point is unstable. This divergent dynamics does not appear when $\phi$ has a finite saturation value.

Spiking dynamics.-The question of whether networks with spiking dynamics exhibit a phase transition to chaos at finite synaptic gain extends beyond the issue of the shape of the $f-I$ curve. In contrast to recent claims [25], we have shown that a sharp transition from a fixed point to chaos in such networks is meaningful only when the synaptic time constant is large, where there is a clear separation between spiking dynamics and rate dynamics. In this limit, depending on the shape of the $f-I$ curve, the underlying rates may be constant in low synaptic gain and become chaotic above a critical gain, similar to the behavior of rate-based dynamics. The general correspondence between smooth rate dynamic models and the dynamics of synaptic currents in neuronal systems with long synaptic time constants has been studied previously [55]. However, the implications on the transition to chaos in random neuronal networks were not previously addressed. In any realistic systems, the time constants are finite; hence, it is important to assess the smoothing of the transition due to finite synaptic times. Here, we have characterized this smoothing by a scaling function with a single crossover exponent. This exponent determines the rate of change from stochastic spiking dynamics with static rates to smooth rate dynamics, as 
the synaptic time constant increases. This scaling analysis predicts relatively strong "finite size" effects of the spiking dynamics near the transition of the corresponding rate dynamics. First, the scaling regime is relatively large, given by $\left|g-g_{c}\right| \propto\left(r \tau_{s}\right)^{-2 / 5}$, where $r$ is the mean firing rate and $\tau_{s}$ is the synaptic integration time. In addition, the effective time constant of the synaptic fluctuations due to spiking scales as $\tau_{\text {eff }} \propto\left(r \tau_{s}\right)^{1 / 5}$. Thus, a sharp transition requires rather large values of $r \tau_{s}$.

Concerning the biologically relevant regime, typical values of mean rates range from an order of $1 \mathrm{~Hz}$ to $100 \mathrm{~Hz}$. Fast synaptic currents (AMPA and $\mathrm{GABA}_{\mathrm{A}}$ ) have decay time constants of the order of 1-10 msec so are not expected to exhibit the above transition. Slow synaptic currents (e.g., NMDA, GABA $A_{B}$, and peptide neurotransmitters) have time constants ranging from a few hundred milliseconds to minutes [40] and thus might be appropriate candidates for exhibiting transition from spike dynamics to rate fluctuations for systems with moderate rates (such as primate visual and motor cortex).

Our analysis of networks of spiking neurons assumed an inhomogeneous Poisson spiking model, which is a wellknown and extensively used statistical model of spiking fluctuations [49,56]. However, it is interesting to ask whether our results hold for deterministic spiking models with an appropriately smooth $f-I$ curve. We believe our results regarding a transition to chaos for large $K$ and large $r \tau_{s}$ are also valid for conductance-based spiking dynamics since they rely only on the separation of time scales between the firing dynamics and synaptic fluctuations. In particular, we expect that for weak synaptic gain, the network will be in an asynchronous state characterized by synaptic inputs and population firing rates whose fluctuation amplitude is small, on the scale of $\tau_{s}$. On the other hand, for strong synaptic gain, these fluctuations will be large even on the scale of $\tau_{s}$, and the statistics of these fluctuation will follow the chaotic dynamics of smooth rate dynamics.

Beyond its simplicity, the advantage of the treatment of the Poisson model is that it demonstrates the condition for rate chaotic fluctuations for stochastically firing neurons as well. Our analysis of the Poisson model was restricted to a threshold-linear rate function. It is straightforward to extend the DMFT equations to a general rate function, including one with a power-law behavior above threshold with $\nu \neq 1$. It will be of interest to study in more detail the effect of fast spiking noise on the network dynamics, particularly for the cases where the (noiseless) $f-I$ curve has $\nu<1 / 2$.

One difference between the Poisson and the deterministic spiking dynamics is the degree of irregularity in the spike times, as measured, e.g., by the standard deviation of the ISI distribution divided by its mean (known as the coefficient of variation). In the Poisson model, the $C V$ is close to 1 in the fixed-point regime (as in the homogeneous
Poisson process) and increases above it in the chaotic regime because of the induced rate fluctuations. In contrast, in deterministic spiking models, the $\mathrm{CV}$ is expected to be substantially lower than 1 in the fixed-point regime. A detailed study of random networks with deterministic spiking dynamics associated with a sufficiently smooth $f-I$ curve and slow synaptic time constants is an interesting topic for future work.

In this work, we have addressed the effect of spiking dynamics in smearing the transition from a state with static underlying synaptic currents to a state where the currents themselves (hence the underlying rates) fluctuate in time. However, there can be other types of transitions from nonchaotic dynamics to chaotic dynamics in a spiking network even for fast synaptic time constants. In the case of deterministic spiking networks, this transition may mark the separation from a limit cycle at low $g$ to a chaotic attractor at high $g$. In this case, the chaotic dynamics typically presents fast time constants, and can not be described as instability in the rates. In addition, it is likely that even in the nonchaotic regime of deterministic spiking models, irregular transients (with an effective negative Lyapunov exponent, termed as stable chaos) will persist for a long time, and convergence time to the limit cycle will grow exponentially with the system size (as in Refs. [5759]). Interestingly, the existence of chaos vs stable chaos in spiking networks has been shown to depend on the details of the spike initiation dynamics [60]. A transition from an irregular but nonchaotic state to a truly chaotic state might be important for the information-processing capacity of the system but will be hard to observe experimentally, as it is not reflected in the properties of the correlation functions.

Our discussion of biological relevance of rate-based dynamics focused on identifying the units in the rate-based models as single neurons and utilized temporal averaging as the mechanism for the adequacy of a rate-based theory. An alternative scenario where the rate description of a spiking network might be applicable is when the system consists of clusters of neurons, where spatial averaging can lead to rate dynamics (e.g., Refs. [53,61,62]). Under this interpretation, single units in our model represent weakly synchronous neuronal subpopulations, and the random connectivity corresponds to the large-scale effective connectivity between these populations. As such, these models can serve as a useful framework for investigating aspects of the large-scale nonlinear dynamics of the nervous system, as measured by EEG and fMRI signals.

This work explored the conditions under which random networks exhibit a transition from fixed point to chaos and the rate of development of chaotic fluctuations near the transition. These questions may bear important consequences for the computations that such networks can produce. Recent models $[13,14,63]$ have shown that random recurrent networks can be shaped through learning to generate a broad repertoire of desired trajectories with 
biologically relevant time scales. These abilities prevail only near the onset of chaos. For substantially low gain, the activity in the network is strongly suppressed, whereas high above the transition, the intrinsic fluctuations are too fast and erratic to match the smooth and slow desired trajectories. It would be very interesting to study in detail how the results of the present work affect the computational powers of recurrent neuronal networks.

\section{ACKNOWLEDGMENTS}

We wish to thank L.F. Abbott and M. Stern for helpful discussions and J.E. Fitzgerald and I. Landau for their comments on the manuscript. We also wish to thank the anonymous reviewers for their helpful insights. This research was supported, in part, by the Gatsby Charitable Foundation, the International Max PlanckHebrew University Center for Sensory Processing of the Brain in Action, the James S. McDonnell Foundation, and the Simons Foundation (SCGB Grant No. 325207 to H. S.).

Note added.-Recently, we became aware of Ref. [64], which partially overlaps with the present work.

\section{APPENDIX A: DMFT EQUATIONS FOR THE AUTOCORRELATIONS}

We extend previous derivations in Ref. [6] to the current model of multiple subpopulation. Here, we show the derivation of the self-consistent dynamical equations for the population-averaged autocorrelation function of the local fields, $\Delta_{k}(\tau)$, defined in Eq. (24). The dynamic equations for the fluctuations in the local fields, $\delta h_{k}^{i}(t)$, are given by Eq. (10), where in the large $N$ limit, the noise terms $\eta_{k}^{i}(\tau)$ are Gaussian random variables with zero mean and $\left\langle\eta_{k}^{i}(t) \eta_{k}^{i}(t+\tau)\right\rangle=\sum_{l} g_{k l}^{2} C_{l}(\tau)$, where $C_{k}(\tau) \equiv$ $\left\langle\phi\left(h_{k}^{i}(t)\right) \phi\left(h_{k}^{i}(t+\tau)\right)\right\rangle$ is calculated self-consistently by integrating over a temporally colored Gaussian noise $\eta_{k}^{i}(\tau)$. A convenient way of treating the temporal correlations between $\delta h_{k}(t)$ and $\delta h_{k}(t+\tau)$ is to introduce three uncorrelated Gaussian variables $y_{1}, y_{2}$, and $z$ with unit variances such that

$$
\begin{aligned}
\delta h_{k}(t) & =\sqrt{\alpha} y_{1}+\sqrt{\beta} z, \\
\delta h_{k}(t+\tau) & =\sqrt{\alpha} y_{2}+\sqrt{\beta} z,
\end{aligned}
$$

with

$$
\begin{aligned}
& \alpha=\Delta_{k}(0)-\Delta_{k}(\tau), \\
& \beta=\Delta_{k}(\tau) .
\end{aligned}
$$

With these variables, the population-averaged autocorrelation function can be written as the integral over independent Gaussian variables,

$$
\begin{aligned}
C_{k}(\tau)= & \int D y_{1} \int D y_{2} \int D z \phi\left(\sqrt{\alpha} y_{1}+\sqrt{\beta} z+u_{k}\right) \\
& \times \phi\left(\sqrt{\alpha} y_{2}+\sqrt{\beta} z+u_{k}\right) .
\end{aligned}
$$

Finally, to yield the self-consistent equations for $\Delta_{k}$, it is convenient to use the Fourier transform of the dynamic equation (10) to get

$$
(1+i \omega) \delta h_{k}^{i}(\omega)=\eta_{k}^{i}(\omega)
$$

from which we obtain

$$
\left(1+\omega^{2}\right)\left\langle\left|\delta h_{k}^{i}(\omega)\right|^{2}\right\rangle=\left\langle\left|\eta_{k}^{i}(\omega)\right|^{2}\right\rangle=\sum_{l} g_{k l}^{2} C_{l}(\omega) .
$$

Finally, performing a Fourier transform back to the time domain, and substituting the result of Eq. (A1), the $P$ selfconsistent equations for the autocorrelations of the local fields read

$$
\begin{aligned}
& \left(1-\frac{\partial^{2}}{\partial \tau^{2}}\right) \Delta_{k}(\tau) \\
& \quad=\sum_{l} g_{k l}^{2}\left\langle\left\langle\phi\left(\sqrt{\Delta_{l}(0)-\Delta_{l}(\tau)} y+\sqrt{\Delta_{l}(\tau)} z+u_{l}\right)\right\rangle_{y}^{2}\right\rangle
\end{aligned}
$$

\section{APPENDIX B: STABILITY EQUATIONS FOR THE FIXED POINTS}

In this Appendix, we derive the stability conditions for the fixed-point states of the network.

\section{Population-average linear response}

For simplicity, we present the derivation with one population architecture. The extension to multiple populations is straightforward.

We evaluate the time-dependent response function

$$
\chi_{i}(t) \equiv \partial \phi\left(h_{i}(t)\right) / \partial h^{0}(0),
$$

and denote by $\chi(t)$ the spatial average of $\chi_{i}$ at the fixedpoint solution. From the equations of motion, we obtain

$$
\begin{aligned}
\left(1+\partial_{t}\right) \chi_{i}(t)= & \phi^{\prime}\left(h_{i}\right) \sum_{j} \mathcal{J}_{i j} \chi_{j}(t)+\phi^{\prime}\left(h_{i}\right) \bar{g} \chi(t) \\
& +\phi^{\prime}\left(h_{i}\right) \delta(t),
\end{aligned}
$$

where $\phi^{\prime}\left(h_{i}\right)$ are the derivatives of the activation function evaluated at the fixed points. Let us consider the general term 
$\phi^{\prime}\left(h_{i}\right) \sum_{j} \mathcal{J}_{i j} \chi_{j}(t)=\frac{\partial}{\partial h(0)} \phi^{\prime}\left(h_{i}\right) \sum_{j} \mathcal{J}_{i j} \phi\left(h_{j}(t)\right)$.

The fixed-point values of $h_{i}$ are independent of a perturbation at time 0. Thus, using Eqs. (10) and (11), we have

$\phi^{\prime}\left(h_{i}\right) \sum_{j} \mathcal{J}_{i j} \chi_{j}(t)=\frac{\partial}{\partial h(0)}\left(1+\partial_{t}\right) \phi^{\prime}\left(h_{i}\right) \delta h_{i}(t)$,

where $\partial_{t}$ stands for the time-derivative operator. After averaging and using mean field theory,

$$
\begin{aligned}
\left\langle\phi^{\prime}\right. & \left.\left(h_{i}\left(t_{2}\right)\right) \delta h_{i}\left(t_{1}\right)\right\rangle \\
= & \left\langle\phi^{\prime}\left(\sqrt{\Delta\left(t_{2}, t_{2}\right)-\Delta\left(t_{2}, t_{1}\right)} x+\sqrt{\Delta\left(t_{2}, t_{1}\right)} z+u\left(t_{2}\right)\right)\right. \\
& \left.\times\left(\sqrt{\Delta\left(t_{1}, t_{1}\right)-\Delta\left(t_{2}, t_{1}\right)} y+\sqrt{\Delta\left(t_{2}, t_{1}\right)} z\right)\right\rangle \\
= & \left\langle\phi ^ { \prime } \left(\sqrt{\Delta\left(t_{2}, t_{2}\right)-\Delta\left(t_{2}, t_{1}\right)} x\right.\right. \\
& \left.\left.+\sqrt{\Delta\left(t_{2}, t_{1}\right)} z+u\left(t_{2}\right)\right) \sqrt{\Delta\left(t_{2}, t_{1}\right)} z\right\rangle \\
= & \Delta\left(t_{2,} t_{1}\right)\left\langle\phi^{\prime \prime}\left(\sqrt{\Delta\left(t_{2}, t_{2}\right)} z+u\left(t_{2}\right)\right)\right\rangle,
\end{aligned}
$$

where $\Delta\left(t, t^{\prime}\right)=\left\langle\delta h(t) \delta h\left(t^{\prime}\right)\right\rangle$. Thus,

$$
\left\langle\phi^{\prime}\left(h_{i}\right) \sum_{j=1}^{N} \mathcal{J}_{i j} \chi_{j}(t)\right\rangle=\left(1+\partial_{t}\right) \frac{\partial \Delta\left(t^{\prime}, t\right)}{\partial h^{0}(0)}\left\langle\phi^{\prime \prime}\right\rangle
$$

with $t^{\prime} \rightarrow \infty$. Finally, we note that in this limit,

$$
\frac{\partial \Delta\left(t_{2, t}\right)}{\partial h^{0}(0)}=\frac{\partial\left\langle\delta h\left(t_{2}\right) \delta h(t)\right\rangle}{\partial h^{0}(0)}
$$

We denote

$$
\chi^{\Delta}(t)=2 \chi^{\Delta}(\infty, t)
$$

where

$$
\chi^{\Delta}\left(t^{\prime}, t\right)=\frac{\partial \Delta\left(t^{\prime}, t\right)}{\partial h^{0}(0)} .
$$

Note that the factor of 2 was introduced because, at the fixed point, we have

$$
\chi^{\Delta}(t, t)=2 \chi^{\Delta}(\infty, t) \equiv \chi^{\Delta}(t)
$$

which is the susceptibility of the mean equal-time variance.

Substituting the above results in Eq. (B2) and averaging, we obtain

$$
\begin{aligned}
(1 & \left.-\left\langle\phi^{\prime}\right\rangle \bar{g}+\partial_{t}\right) \chi(t) \\
& =\frac{1}{2}\left\langle\phi^{\prime \prime}\right\rangle\left(1+\partial_{t}\right) \chi^{\Delta}(t)+\left\langle\phi^{\prime}\right\rangle \delta(t) .
\end{aligned}
$$

Calculating of $\chi^{\Delta}$.-From the DMFT, we obtain

$$
\left(1+\partial_{t_{1}}\right)\left(1+\partial_{t_{2}}\right) \Delta\left(t_{2}, t_{1}\right)=g^{2} C\left(t_{1}, t_{2}\right),
$$

where

$$
\begin{aligned}
C\left(t_{1}, t_{2}\right)= & \left\langle\phi \left(\sqrt{\Delta\left(t_{2}, t_{2}\right)-\Delta\left(t_{2}, t_{1}\right)} x\right.\right. \\
& \left.+\sqrt{\Delta\left(t_{2}, t_{1}\right)} z+u\left(t_{2}\right)\right) \phi\left(\sqrt{\Delta\left(t_{1}, t_{1}\right)-\Delta\left(t_{2}, t_{1}\right)} y\right. \\
& \left.\left.+\sqrt{\Delta\left(t_{2}, t_{1}\right)} z+u\left(t_{1}\right)\right)\right\rangle .
\end{aligned}
$$

Thus,

$$
\begin{aligned}
(1+ & \left.\partial_{t_{1}}\right)\left(1+\partial_{t_{2}}\right) \chi^{\Delta}\left(t_{2,}, t_{1}\right) \\
= & g^{2}\left[\partial C\left(t_{1}, t_{2}\right) / \partial \Delta\left(t_{2}, t_{1}\right) \chi^{\Delta}\left(t_{2}, t_{1}\right)\right. \\
& +\partial C\left(t_{1}, t_{2}\right) / \partial \Delta\left(t_{2}, t_{2}\right) \chi^{\Delta}\left(t_{2}, t_{2}\right) \\
& +\partial C\left(t_{1}, t_{2}\right) / \partial \Delta\left(t_{1}, t_{1}\right) \chi^{\Delta}\left(t_{1}, t_{1}\right) \\
& +\left\langle\phi^{\prime}\left(t_{2}\right) \phi\left(t_{1}\right)\right\rangle\left[\bar{g} \chi\left(t_{2}\right)+\delta\left(t_{2}\right)\right] \\
& \left.+\left\langle\phi\left(t_{2}\right) \phi^{\prime}\left(t_{1}\right)\right\rangle\left[\bar{g} \chi\left(t_{1}\right)+\delta\left(t_{1}\right)\right]\right],
\end{aligned}
$$

where, by derivation of Eq. (14),

$$
\partial C\left(t_{1}, t_{2}\right) / \partial \Delta\left(t_{2}, t_{1}\right)=\left\langle\phi^{\prime}\left(t_{2}\right) \phi^{\prime}\left(t_{1}\right)\right\rangle,
$$

$$
\partial C\left(t_{1}, t_{2}\right) / \partial \Delta\left(t_{1}, t_{1}\right)=\frac{1}{2}\left\langle\phi\left(t_{1}\right) \phi^{\prime \prime}\left(t_{1}\right)\right\rangle,
$$

and

$$
\frac{\partial u}{\partial h^{0}}=\bar{g} \chi(t)+\delta(t)
$$

We assume that $t_{2}>t_{1}>t_{0}$ are all large, but their difference is of order 1 .

Let us take $t_{2}-t_{1} \rightarrow \infty$. Then, substituting Eq. (B10), one obtains

$$
\begin{aligned}
\left(1+\partial_{t}\right) \chi^{\Delta}(t)= & g^{2}\left[\left\langle\phi^{\prime 2}\right\rangle+\left\langle\phi \phi^{\prime \prime}\right\rangle\right] \chi^{\Delta}(t) \\
& +2 g^{2}\left\langle\phi \phi^{\prime}\right\rangle[\bar{g} \chi(t)+\delta(t)] .
\end{aligned}
$$

Equations (B11) and (B18) constitute two coupled equations for the response functions of the mean and variance of the population activity. It is perhaps convenient to eliminate the time derivative in the right-hand side of Eq. (B11) by substituting Eq. (B18), resulting in 


$$
\begin{aligned}
\left(1-\left\langle\phi^{\prime}\right\rangle \bar{g}+\partial_{t}\right) \chi(t)= & \frac{1}{2}\left\langle\phi^{\prime \prime}\right\rangle g^{2}\left[\left\langle\phi^{\prime 2}\right\rangle+\left\langle\phi \phi^{\prime \prime}\right\rangle\right] \chi^{\Delta}(t) \\
& +\left\langle\phi^{\prime \prime}\right\rangle g^{2}\left\langle\phi \phi^{\prime}\right\rangle[\bar{g} \chi(t)+\delta(t)] \\
& +\left\langle\phi^{\prime}\right\rangle \delta(t) .
\end{aligned}
$$

We can write these equations as

$$
\begin{gathered}
\left(a+\partial_{t}\right) \chi(t)=b \chi(t)+c \delta(t), \\
\left(d+\partial_{t}\right) \chi^{\Delta}(t)=e \chi(t)+f \delta(t),
\end{gathered}
$$

or, in Fourier and matrix representation,

$$
\left[\begin{array}{cc}
(1-\bar{g} a+i \omega) & -b \\
-\bar{g} e & (1-d+i \omega)
\end{array}\right]\left[\begin{array}{c}
\chi \\
\chi^{\Delta}
\end{array}\right]=\left[\begin{array}{l}
a \\
e
\end{array}\right],
$$

where

$$
\begin{aligned}
& a=\left\langle\phi^{\prime \prime}\right\rangle g^{2}\left\langle\phi \phi^{\prime}\right\rangle+\left\langle\phi^{\prime}\right\rangle, \\
& d=g^{2}\left[\left\langle\phi^{\prime 2}\right\rangle+\left\langle\phi \phi^{\prime \prime}\right\rangle\right], \\
& b=\frac{1}{2}\left\langle\phi^{\prime \prime}\right\rangle g^{2}\left[\left\langle\phi^{\prime 2}\right\rangle+\left\langle\phi \phi^{\prime \prime}\right\rangle\right],
\end{aligned}
$$

and

$$
e=2 g^{2}\left\langle\phi \phi^{\prime}\right\rangle \bar{g}
$$

Multiple population network.-A straightforward generalization to the case of multiple populations yields

$$
\chi_{k l}(t) \equiv \frac{\partial m_{k}(t)}{\partial h_{l}(0)}
$$

and

$$
\chi_{k l}^{\Delta}(t)=\frac{\partial}{\partial h_{l}(0)} \Delta_{k}(t)
$$

In this case, the equation for $\chi^{\Delta}(t)$ [Eq. (B18)] reads

$$
\begin{aligned}
\left(1+\partial_{t}\right) \chi_{k l}^{\Delta}(t)= & \sum_{m} g_{k m}^{2}\left[\left\langle\phi_{m}^{\prime 2}\right\rangle+\left\langle\phi_{m} \phi_{m}^{\prime \prime}\right\rangle\right] \chi_{m l}^{\Delta}(t) \\
& +2 \sum_{m} g_{k m}^{2}\left\langle\phi_{m} \phi_{m}^{\prime}\right\rangle \\
& \times\left[\sum_{m^{\prime}} \bar{g}_{m m^{\prime}} \chi_{m^{\prime} l}(t)+\delta_{m l} \delta(t)\right],
\end{aligned}
$$

or, in matrix notation,

$$
\left(\mathbf{I}-\mathbf{D}+\partial_{t}\right) \chi^{\Delta}=\mathbf{E}(\overline{\mathbf{g}} \chi+\mathbf{I} \delta(t)) .
$$

Likewise, the dynamical equation on $\chi(t)$ is

$$
\begin{aligned}
\left(1+\partial_{t}\right) \chi_{k l}(t)= & \sum_{m} \bar{g}_{k m}\left\langle\phi_{m}^{\prime}\right\rangle \chi_{m l}(t)+\left\langle\phi_{k}^{\prime}\right\rangle \delta_{k l} \delta(t) \\
& +\frac{1}{2}\left\langle\phi_{k}^{\prime \prime}\right\rangle\left(1+\partial_{t}\right) \chi_{k l}^{\Delta}(t)
\end{aligned}
$$

or

$$
\left(\mathbf{I}-\mathbf{A} \overline{\mathbf{g}}+\partial_{t}\right) \chi(t)=\mathbf{B} \chi^{\Delta}+\mathbf{A} \delta(t) .
$$

In the above, we have defined the $P \times P$ matrices

$$
\begin{aligned}
& A_{k l}=\delta_{k l}\left\langle\phi_{l}^{\prime}\right\rangle+\left\langle\phi_{k}^{\prime \prime}\right\rangle g_{k l}^{2}\left\langle\phi_{l} \phi_{l}{ }^{\prime}\right\rangle, \\
& B_{k l}=\frac{1}{2} g_{k l}^{2}\left\langle\phi_{k}^{\prime \prime}\right\rangle\left[\left\langle\phi_{l}^{\prime 2}\right\rangle+\left\langle\phi_{l} \phi_{l}^{\prime \prime}\right\rangle\right], \\
& D_{k l}=g_{k l}^{2}\left[\left\langle\phi_{l}^{\prime 2}\right\rangle+\left\langle\phi_{l} \phi_{l}^{\prime \prime}\right\rangle\right],
\end{aligned}
$$

and

$$
E_{k l} \equiv 2 g_{k l}^{2}\left\langle\phi_{l} \phi_{l}{ }^{\prime}\right\rangle
$$

Equations (B30) and (B32) can be written using a $2 P \times 2 P$ matrix in Fourier space as

$$
\left[\begin{array}{cc}
(\mathbf{I}-\mathbf{A} \overline{\mathbf{g}}+i \omega) & -\mathbf{B} \\
-\mathbf{E} \overline{\mathbf{g}} & (\mathbf{I}-\mathbf{D}+i \omega)
\end{array}\right]\left[\begin{array}{c}
\chi(\omega) \\
\chi^{\Delta}(\omega)
\end{array}\right]=\left[\begin{array}{l}
\mathbf{A} \\
\mathbf{E}
\end{array}\right]
$$

Thus, the fixed point is stable against population-average perturbations provided that all the eigenvalues of the matrix

$$
\left[\begin{array}{cc}
\mathbf{I}-\mathbf{A} \overline{\mathbf{g}} & -\mathbf{B} \\
-\mathbf{E} \overline{\mathbf{g}} & 1-\mathbf{D}
\end{array}\right]
$$

have a negative real part.

\section{Response to local perturbations}

The susceptibility matrix of the network to a random local perturbation is defined as

$$
\chi_{k l}^{i j}\left(t-t^{\prime}\right)=\partial h_{k}^{i}(t) / \partial h_{l}^{0 j}\left(t^{\prime}\right),
$$

where $h_{l}^{0 j}\left(t^{\prime}\right)$ are infinitesimal external perturbations around the fixed-point state. We are interested in the mean square of this quantity. Using the dynamical equation (2), we find the time evolution of the susceptibility 


$$
\begin{aligned}
\left(1+\frac{d}{d t}\right) \chi_{k l}^{i j}\left(t-t^{\prime}\right)= & \sum_{l^{\prime}=1}^{P} \sum_{j^{\prime}=1}^{N} \mathcal{J}_{k l^{\prime}}^{i j^{\prime}} \phi^{\prime}\left(h_{l^{\prime}}^{j^{\prime}}\right) \chi_{l^{\prime} l}^{j^{\prime} j}\left(t-t^{\prime}\right) \\
& +\frac{1}{N} \sum_{l^{\prime}=1}^{P} \bar{g}_{k l^{\prime}} \sum_{j^{\prime}} \phi^{\prime}\left(h_{l^{\prime}}^{j^{\prime}}\right) \chi_{l^{\prime} l}^{j^{\prime} j}\left(t-t^{\prime}\right) \\
& +\delta\left(t-t^{\prime}\right) \delta_{i j} \delta_{k l}, \quad t \geq 0, \quad \text { (B }
\end{aligned}
$$

where $\delta_{i j}$ and $\delta(t)$ are the Kronecker and Dirac delta functions, respectively. Defining

$$
G_{k l}\left(\omega_{1,}, \omega_{2}\right)=N^{-1} \sum_{i j}\left\langle\chi_{k l}^{i j}\left(\omega_{1}\right) \chi_{k l}^{i j}\left(\omega_{2}\right)\right\rangle
$$

and averaging Eq. (B40) yields

$$
\mathbf{G}\left(\omega_{1,}, \omega_{2}\right)=\left[\left(1+i \omega_{1}\right)\left(1+i \omega_{2}\right) I-\mathbf{M}\right]^{-1},
$$

where $\mathbf{M}$ is the stability matrix, $M_{k l}=\left\langle\phi^{\prime}\left(h_{k}^{i}\right) \phi^{\prime}\left(h_{l}^{i}\right)\right\rangle$ [Eq. (23)].

Note that $G_{k l}$ are of order 1 ; the contribution of the uniform $\chi_{k l}$ to $G_{k l}$ is of order $1 / N$ and hence negligible. Furthermore, the uniform components of the interactions [in Eq. (B40)] do not contribute to Eq. (B41) to leading order, as they are smaller by a factor of $1 / N$ relative to the random contributions.

\section{APPENDIX C: STABILITY EQUATIONS AND THE LARGEST LYAPUNOV EXPONENT}

The susceptibility matrix of the network to a random local perturbation is defined as

$$
\chi_{k l}^{i j}\left(t, t^{\prime}\right)=\partial h_{k}^{i}(t) / \partial h_{l}^{0 j}\left(t^{\prime}\right),
$$

where $h_{l}^{0 j}\left(t^{\prime}\right)$ are infinitesimal external perturbations. Using the dynamical equation (2), we find the time evolution of the susceptibility,

$$
\begin{aligned}
\left(1+\frac{d}{d t}\right) \chi_{k l}^{i j}\left(t, t^{\prime}\right)= & \sum_{l^{\prime}=1}^{P} \sum_{j^{\prime}=1}^{N} \mathcal{J}_{k l^{\prime}}^{i j^{\prime}} \phi^{\prime}\left(h_{l^{\prime}}^{j^{\prime}}(t)\right) \chi_{l^{\prime} l}^{j^{\prime} j}\left(t, t^{\prime}\right) \\
& +\frac{1}{N} \sum_{l^{\prime}=1}^{P} \bar{g}_{k l^{\prime}} \sum_{j^{\prime}} \phi^{\prime}\left(h_{l^{\prime}}^{j^{\prime}}(t)\right) \chi_{l^{\prime} l}^{j^{\prime} j}\left(t, t^{\prime}\right) \\
& +\delta\left(t-t^{\prime}\right) \delta_{i j} \delta_{k l}, \quad t \geq 0, \quad
\end{aligned}
$$

where $\delta_{i j}$ and $\delta(t)$ are the Kronecker and Dirac delta functions, respectively, denoting a local spatiotemporal perturbation.

Population averaged susceptibility. - Let us define $\chi^{0}$ as the within-population spatial-average susceptibility $\chi_{k l}^{0}\left(t-t^{\prime}\right)=\frac{1}{N^{2}} \sum_{i, j=1}^{N} \chi_{k l}^{i j}\left(t, t^{\prime}\right)=\frac{1}{N^{2}} \sum_{i, j=1}^{N}\left\langle\chi_{k l}^{i j}\left(t, t^{\prime}\right)\right\rangle_{\mathcal{J}}$.

This uniform matrix is formally given by

$\chi^{0}\left(t-t^{\prime}\right)=\left\langle\left[I-\tilde{\mathcal{J}}-\frac{1}{N} \tilde{J}^{0}\right]^{-1}\right\rangle a(t) \delta\left(t-t^{\prime}\right), \quad t \geq t^{\prime}$,

where $I$ is the spatial identity matrix, $a(t)$ is the temporal operator $a=(1+d / d t)^{-1}, \tilde{\mathcal{J}}_{k l}^{i j}=a \mathcal{J}_{k l}^{i j} \phi^{\prime}\left(h_{l^{\prime}}^{j^{\prime}}\right)$, and $\tilde{J}_{k l}^{0 i j}=$ $a \bar{g}_{k l} \phi^{\prime}\left(h_{l}^{j}\right)$. (The right-hand side is formally an $N P \times N P$ matrix; however, it is meant to be reduced to a $P \times P$ matrix by averaging over the $i, j$ indices.)

Expanding the right-hand side in powers of $\tilde{\mathcal{J}}$ shows that all contributions vanish upon averaging; hence,

$$
\chi^{0}\left(t-t^{\prime}\right)=\left\langle\left[I-\frac{1}{N} \tilde{J}^{0}\right]^{-1}\right\rangle a(t) \delta\left(t-t^{\prime}\right), \quad t \geq t^{\prime} .
$$

Furthermore, performing the averaging in the right-hand side of Eq. (C5) and Fourier transforming the result yields

$$
\chi^{0}(\omega)=\frac{1}{N}\left[(1+i \omega) I-J^{0}\right]^{-1}
$$

where

$$
J_{k l}^{0}=\bar{g}_{k l}\left\langle\phi^{\prime}\left(h_{l}^{i}\right)\right\rangle .
$$

Note that this population-averaged susceptibility is the solution of the population-averaged part of the dynamics, namely,

$$
\left(1+\frac{d}{d t}\right) \chi_{k l}^{0}(t)=\sum_{k^{\prime}} \bar{g}_{k k^{\prime}}\left\langle\phi^{\prime}\left(h_{i}^{l}\right)\right\rangle \chi_{k^{\prime} l}^{0}(t)+\frac{1}{N} \delta_{k l} \delta(t), \quad t \geq 0 .
$$

Note that all elements of $\chi_{k l}^{0}$ are of order $1 / N$, including the diagonal. This is because $\chi_{k k}^{0 i i}$, which is of order 1 , adds only a $1 / N$ contribution to the spatially averaged quantity, Eq. (C3).

Statistics of the full susceptibility.-We now evaluate the full susceptibility, $\chi_{k l}^{i j}$. The random fluctuations in the susceptibility, i.e., the off-diagonal elements of $\chi$, are of order $1 / \sqrt{N}$ as will be seen below; i.e., locally they are much larger than $\chi_{k l}^{0}$. Their average second-order moments are defined in terms of

$$
G_{k l}\left(t_{a}, t_{b}, t_{c}, t_{d}\right)=\frac{1}{N} \sum_{i, j}^{N}\left\langle\chi_{k l}^{i j}\left(t_{a}, t_{c}\right) \chi_{k l}^{i j}\left(t_{b}, t_{d}\right\rangle_{\mathcal{J}} .\right.
$$


To evaluate Eq. (C9), we multiply two realizations of Eq. (C2) (differing in the time indices) and take the spatial average over all neurons, leading to

$$
\begin{aligned}
& \left(1+\frac{\partial}{\partial t_{a}}\right)\left(1+\frac{\partial}{\partial t_{b}}\right) G_{k l}\left(t_{a}, t_{b}, t_{c}, t_{d}\right) \\
& -\sum_{m} M_{k l}\left(t_{a}-t_{b}\right) G_{m l}\left(t_{a}, t_{b}, t_{c}, t_{d}\right) \\
& \quad=\delta\left(t_{a}-t_{b}-t_{c}+t_{d}\right) \delta\left(t_{a}+t_{b}-t_{c}-t_{d}\right) \delta_{k l},
\end{aligned}
$$

where

$$
M_{k l}(\tau)=g_{k l}^{2}\left\langle\phi ^ { \prime } \left( h_{i}^{l}(t+\tau) \phi^{\prime}\left(h_{i}^{l}(t)\right\rangle .\right.\right.
$$

Note that $G_{k l}$ are of order 1, while the contribution of $\chi_{k l}^{0}$ to them is of order $1 / N$ and hence negligible. Furthermore, the uniform components of the interactions [in Eq. (C2)] do not contribute to Eq. (C9) to leading order as they are smaller by a factor of $1 / N$ relative to the random contributions.

Defining new time variables as $\tau=t_{a}-t_{b}, \tau^{\prime}=t_{c}-t_{d}$, $T=t_{a}+t_{b}$, and $T^{\prime}=t_{c}+t_{d}$, Eq. (C10) can be written as

$$
\begin{aligned}
& {\left[\left(\left(1+\frac{\partial}{\partial T}\right)^{2}-1\right) I+\mathcal{H}(\tau)\right] G} \\
& \quad=2 \delta\left(T-T^{\prime}\right) \delta\left(\tau-\tau^{\prime}\right) \mathbf{I}
\end{aligned}
$$

where $\mathcal{H}(\tau)$ is the $P \times P$ operator [see Eq. (29)],

$$
\mathcal{H}(\tau)=-\frac{\partial^{2}}{\partial \tau^{2}} \mathbf{I}+\mathbf{I}-\mathbf{M}(\tau)
$$

Fixed point.-At the fixed point,

$$
\mathcal{H}(\tau)=-\frac{\partial^{2}}{\partial \tau^{2}} \mathbf{I}+\mathbf{I}-\mathbf{M},
$$

where $M$ is a time-independent $P \times P$ matrix. Fourier transforming Eq. (C12) yields

$$
\left[\left(2 i \Omega-\Omega^{2}\right) I+\left(\omega^{2}+1\right) I-M\right] G(\Omega, \omega)=4 \pi \mathbf{I},
$$

where $\Omega$ and $\omega$ are the frequencies associated with $T$ and $\tau$, respectively, and

$$
\begin{aligned}
G(\Omega, \omega)= & \int d T \int d \tau G(T, 0, \tau, 0) \\
& \times \exp (-i \Omega T-i \omega \tau) .
\end{aligned}
$$

Thus, the zero-frequency limit yields a static matrix given (up to a constant) by

$$
\mathbf{G}(\Omega=0, \omega=0)=(\mathbf{I}-\mathbf{M})^{-1},
$$

where $\mathbf{M}$ is defined in Eq. (30) and fixed-point stability requires that all eigenvalues of $M$ have real parts less than 1 . Note that $\mathbf{G}_{k l}=N\left\langle\left(\chi_{k l}^{i j}(\omega=0)\right)^{2}\right\rangle$, i.e., the square average of the local static susceptibility.

Chaotic regime.-The largest Lyapunov exponent is the exponential divergent rate of the squared susceptibility $N\left\langle\left(\chi_{k l}^{i j}(t, 0)\right)^{2}\right\rangle=G_{k l}(2 t, 0,0,0)$, in the chaotic state, which corresponds to $T=2 t$, and $T^{\prime}=\tau=\tau^{\prime}=0$. Thus, the largest Lyapunov exponent is found by

$$
\lambda_{L}=\lim _{t \rightarrow \infty} \frac{1}{2 t} \ln \sum_{k l}^{P} G_{k l}(2 t, 0,0,0) .
$$

To evaluate it, we first write the time-dependent solution of Eq. (C12) as

$G_{k l}\left(T, T^{\prime}, \tau, \tau^{\prime}\right)=\sum_{\mu=1}^{\infty} \Gamma_{\mu}\left(T, T^{\prime}\right)\left|\psi_{k}^{\mu}(\tau)\right\rangle\left\langle\psi_{l}^{\mu}\left(\tau^{\prime}\right)\right|$,

where $\left\langle\psi^{\mu}(\tau)\right|$ and $\left.\mid \psi^{\mu}(\tau\rangle\right)$ are the left and right $\mu$ th eigenvectors of the Hamiltonian $\mathcal{H}(\tau)$. We note that, in general, $\mathcal{H}(\tau)$ is non-Hermitic and the eigenvectors $\left|\psi^{\mu}(\tau)\right\rangle$ are not necessarily orthogonal. From Eq. (C12), we find that the general solution for $\Gamma_{\mu}\left(T, T^{\prime}\right)$ is proportional to $e^{\lambda_{\mu}\left(T-T^{\prime}\right)}$ with

$$
\lambda_{\mu}=-1 \pm \sqrt{1-\epsilon_{\mu}}
$$

where $\epsilon_{\mu}$ is the corresponding eigenvalue of $\mathcal{H}(\tau)$. For completeness, one must consider the boundary conditions on the solutions, namely, that a solution exists only for $T>T^{\prime}$, and that the second derivative of $\mathbf{G}$ is a delta function while the first derivative is finite. One obtains

$$
\begin{aligned}
G_{k l}\left(T, T^{\prime}, \tau, \tau^{\prime}\right)= & 4 \sum_{\mu=1}^{\infty} \frac{\Theta\left(T-T^{\prime}\right)\left|\psi_{k}^{\mu}(\tau)\right\rangle\left\langle\psi_{l}^{\mu}\left(\tau^{\prime}\right)\right|}{\sqrt{1-\epsilon_{\mu}}} \\
& \times e^{-\left(T-T^{\prime}\right)} \sinh \left(\left(T-T^{\prime}\right) \sqrt{1-\epsilon_{\mu}}\right) .
\end{aligned}
$$

Finally,

$$
\begin{aligned}
N\left\langle\chi_{k l}^{2}(t, 0)\right\rangle= & G_{k l}(2 t, 0,0,0) \\
= & 4 \sum_{\mu=1}^{\infty} \frac{\left|\psi_{k}^{\mu}(0)\right\rangle\left\langle\psi_{l}^{\mu}(0)\right|}{\sqrt{1-\epsilon_{\mu}}} \\
& \times e^{-2 t} \sinh \left(2 t \sqrt{1-\epsilon_{\mu}}\right) .
\end{aligned}
$$

Thus, the maximal Lyapunov exponent is given by

$$
\lambda_{L}=-1+\sqrt{1-\epsilon_{0}},
$$

where $\epsilon_{0}$ is the ground state of the Hamiltonian (29). 


\section{APPENDIX D: EXPONENTIAL TRANSFER FUNCTION}

We show in the following that a single inhibitory population, with the architecture studied in Sec. III and an exponential transfer function, $\phi(x)=e^{x}$, does not exhibit a chaotic phase. The fixed point of the dynamic is stable when $g^{2} C^{\prime}(x)<1$, where $C=\left\langle\phi_{i}^{2}\right\rangle$, leading to

$$
g^{2}\left\langle\exp \left(\sqrt{\Delta_{0}} z+u\right)\right\rangle=g^{2} e^{2 u+\Delta_{0}}=g^{2} m^{2}<1 .
$$

The variance, given by $\Delta_{0}=g^{2} C(x)$, is

$\Delta_{0}=g^{2}\left\langle\exp \left(2 \sqrt{\Delta_{0} z}+2 u\right)\right\rangle=g^{2} e^{2 u+\Delta_{0}}=g^{2} m^{2}$.

It follows from Eqs. (D1) and (D2) that the fixed point loses its stability at the critical point $g_{c}$ where $\Delta_{0}=g^{2} m^{2}=1$. For variance values higher than 1 , the dynamics is characterized by the autocorrelation function given by the solution to the dynamical MF differential equation in Eq. (45). A stable chaotic solution requires

$$
\left.\frac{\partial^{2}}{\partial \tau^{2}} \Delta\right|_{\tau=\infty}=0
$$

or, equivalently, the vanishing of the potential (46) at $\tau=\infty$. Carrying the Gaussian integrals in Eq. (46), one finds that if the potential vanishes, then

$$
\Delta_{0}=\Delta(\infty) e^{3 \Delta(\infty)}
$$

Since every solution for the autocorrelation function requires $0 \leq \Delta(\infty) \leq \Delta_{0}$, Eq. (D4) cannot be obeyed for a nonvanishing variance, and a chaotic phase does not exist. Consequently, when $\Delta_{0}>1$, the mean activity diverges.

\section{APPENDIX E: DYNAMIC EQUATION NEAR CRITICALITY}

Single population.-Using the homogeneity of the transfer function, the correlation function $C(\tau)$ in Eq. (26) can be written in terms of the parameter $q(\tau)=1-\Delta(\tau) / \Delta_{0}$ giving

$C(\tau)=\Delta_{0}^{2 \nu}\left\langle\langle\phi(\sqrt{q(\tau)} y+\sqrt{1-q(\tau)} z+x)\rangle_{y}^{2}\right\rangle_{z}$.

Near and above criticality, $C(\tau)$ can be expanded in powers of $q(\tau)$ about the fixed-point value $q(\tau)=0$. Since linear analysis is not sufficient to account for the critical phenomena, we keep all terms up to the first nonlinear term in $q(\tau)$,

$\Delta_{0}^{-2 \nu} C=\left\langle\phi_{\nu}^{2}(z+x)\right\rangle-\nu^{2}\left\langle\phi_{\nu-1}^{2}(z+x)\right\rangle q+c q^{\rho}$,

where $c$ is a numerical factor of order 1 . The Gaussian integral in $\left\langle\phi_{\nu-n}^{2}\right\rangle$ diverges for $2(\nu-n) \leq-1$. As a result, the first nonlinear term may be larger than quadratic. The first nonlinear term is $\rho=2$ for $\nu>\frac{3}{2}$ and $\rho=\frac{3}{2}$ for $\frac{1}{2}<\nu \leq \frac{3}{2}$.

For convenience, we redefine the gain parameter as $\tilde{g}=g \Delta_{0}^{(\nu-1) / 2}$; the value of the gain $g$ can be recovered using the mean field equations (36). The dynamic equation (45) can be written as

$$
\begin{aligned}
\frac{\partial^{2}}{\partial \tau^{2}} q= & \tilde{g}^{2}\left\langle\phi_{\nu}^{2}(z+x)\right\rangle-1 \\
& +\left(1-\tilde{g}^{2} \nu^{2}\left\langle\phi_{\nu-1}^{2}(z+x)\right\rangle\right) q+\tilde{g}^{2} c q^{\rho} .
\end{aligned}
$$

Next, we expand each term in Eq. (E3) in powers of $\epsilon=g^{2} / g_{c}^{2}-1$, which controls the distance from criticality. We also denote $\delta x=x-x_{c}$, which is a function of $\epsilon$. Equation (E3) can be written as

$$
\frac{\partial^{2} q(\tau)}{\partial \tau^{2}}=a(\epsilon)+b(\epsilon) q(\tau)+c q^{\rho}(\tau)
$$

where

$$
\begin{aligned}
a(\epsilon)= & \epsilon+2 \nu \tilde{g}_{c}^{2}\left\langle\left[z+x_{c}\right]_{+}^{2 \nu-1}\right\rangle \delta x(1+\epsilon) \\
& +2 \nu(2 \nu-1) \tilde{g}_{c}^{2}\left\langle\left[z+x_{c}\right]_{+}^{2 \nu-2}\right\rangle \delta x^{2}+\mathcal{O}\left(\epsilon \delta x^{2}\right)
\end{aligned}
$$

and

$$
b(\epsilon)=\epsilon+2 \tilde{g}^{2} \nu^{2} b_{\nu}^{(1)} \delta x .
$$

The factor $b_{\nu}^{(1)}$ is of order 1 and equals

$$
b_{\nu}^{(1)}= \begin{cases}(\nu-1)\left\langle\left[z+x_{c}\right]_{+}^{2 \nu-3}\right\rangle & \nu>1 \\ \frac{1}{\sqrt{2 \pi}} & \nu=1 \\ \left(\left\langle\left[z+x_{c}\right]_{+}^{2 \nu-1}\right\rangle+\left\langle\left[z+x_{c}\right]_{+}^{2 \nu-2}\right\rangle\right) & \frac{1}{2}<\nu<1 .\end{cases}
$$

In Eqs. (E5)-(E7), we have used the stability condition (22),

$$
\nu^{2} \tilde{g}_{c}^{2}\left\langle\phi_{\nu-1}^{2}\left(z+x_{c}\right)\right\rangle=1,
$$

and the fixed-point solution for the variance, which also holds at the transition point, Eq. (12),

$$
\tilde{g}_{c}^{2}\left\langle\phi_{\nu}^{2}\left(z+x_{c}\right)\right\rangle=1 \text {. }
$$

We expect that $a(\epsilon)$ would scale as $b(\epsilon) q$, implying that the leading order in Eq. (E5) vanishes and

$$
\delta x=-\frac{1}{2 \nu} \tilde{g}_{c}^{-2}\left\langle\left[z+x_{c}\right]_{+}^{2 \nu-1}\right\rangle^{-1} \epsilon .
$$


Thus, we can write $b(\epsilon)=\epsilon b$. Following the same argument as in Eq. (46), we define a classical potential for $q(\tau)$ by integrating the left-hand side of Eq. (E4), giving

$$
V[q]=a(\epsilon) q+\frac{1}{2} \epsilon b q^{2}+\frac{c}{\rho+1} q^{\rho+1} .
$$

By applying the boundary requirements for a stable chaotic solution, $V[0]=V[q(\infty)]$ and $\left.(d V / d q)\right|_{\tau=\infty}=0$, one gets the scaling of $q$ and $a$ for small $\epsilon$,

$$
q(\infty)=\left(\frac{(1+\rho)}{2 \rho} \frac{b}{c} \epsilon\right)^{1 /(\rho-1)}
$$

and

$$
a=-\frac{\rho-1}{\rho+1} c q^{\rho}(\infty) \sim \epsilon^{\rho /(\rho-1)} .
$$

Multiple populations.-In a network composed of $P$ populations, stability is determined by the eigenvalues of the $P \times P$ stability matrix, Eq. (29). Here, we define a normalized stability matrix $\tilde{M}_{k l}=\Delta_{k}^{-1}(0) M_{k l}=$ $\tilde{g}_{k l}^{2}\left\langle\phi^{\prime 2}\left(z+x_{k}\right\rangle\right.$ and

$$
\tilde{g}_{k l}^{2}=g_{k l}^{2} \frac{\Delta_{l}(0)}{\Delta_{k}(0)}
$$

We write $\tilde{M}_{k l}=\sum_{\mu} \Lambda_{\mu} R_{k} L_{l}^{*}$, where $\Lambda_{\mu}$ are the eigenvalues ordered according to a decreasing value of their real part, and $R, L$ are the right and left eigenvectors of $\tilde{\mathbf{M}}$, respectively. At the transition, $\Lambda_{1}=1$ and $\operatorname{Re} \Lambda_{\mu \neq 1} \leq 1$. When $g_{k l} \approx g_{k l}^{c}$, the unstable eigenvalue is $\Lambda_{1}=1+\epsilon$, where $|\epsilon| \ll 1$ and the sign of $\epsilon$ determines which direction in the space of $g$ (away from $g_{k l}^{c}$ ) is the unstable one. To leading order, the eigenvector $R_{1}$ does not change. Note that $\Lambda_{1}$ depends on both $g$ and $x$. For convenience, we define $\epsilon$ via $\Lambda_{1}\left(g, x_{c}\right)$, i.e., as the change induced in $\Lambda_{1}$ due to the change in $g$ for $x=x_{c}$. We are interested in the properties of the system for $0<\epsilon \ll 1$. For multiple subpopulation, Eq. (E4) takes the form

$$
\frac{\partial^{2} q_{k}}{\partial \tau^{2}}=a_{k}(\epsilon)+\sum_{k l}(\mathbf{I}-\tilde{\mathbf{M}})_{k l} q_{l}+\sum \tilde{g}_{k l}^{2} c_{l} q_{l}^{\rho},
$$

where $a_{k}=1-\sum_{l} \tilde{g}_{k l}^{2} C\left\langle\phi^{2}\left(z+x_{k}\right\rangle\right.$, which vanishes at criticality because of Eq. (12), and $\tilde{M}_{k l}=\tilde{g}_{k l}^{2}\left\langle\phi^{\prime 2}\left(z+x_{k}\right\rangle\right.$. Since the matrix $\mathbf{I}-\tilde{\mathbf{M}}$ vanishes only in the 1 direction, the dominant component of the vector $q$ is in this direction. To see this, we make the ansatz $q_{k}(\tau) \equiv 1-\Delta_{k}(\tau) / \Delta_{k}^{0}=$ $\sum_{\mu} \zeta^{\mu}(\tau) R_{\mu}^{k}$ and assume that $\left|\zeta^{1}\right| \gg\left|\zeta^{\mu}\right|$. We then obtain $\frac{\partial^{2} \zeta^{1}}{\partial \tau^{2}}=\hat{a}^{1}(\epsilon)+\left(1-\Lambda_{1}\right) \zeta^{1}(\tau)+\hat{c}^{1} \zeta^{1}(\tau)^{\rho}$,

$\frac{\partial^{2} \zeta^{\mu}}{\partial \tau^{2}}=\hat{a}^{\mu}(\epsilon)+\left(1-\Lambda_{\mu}\right) \zeta^{1}(\tau)+\hat{c}^{\mu} \zeta^{1}(\tau)^{\rho} \mu \neq 1$,

where $\hat{a}$ and $\hat{c}$ are the factors in the basis of $\tilde{\mathbf{M}}$. Here, $1-\Lambda_{1}$ vanishes at the transition to the order of $\epsilon, \delta x$, while $\left(1-\Lambda_{\mu}\right)$ remains of order 1 near the transition. Thus, $\zeta^{\mu}(\tau)$ scales as $\zeta^{1}(\tau)^{\rho} \ll \zeta(\tau)$, and the "normal form" of the dynamics of $q_{k}$, Eq. (E15), is the same as that of a single population, Eq. (E4). In addition, in the generic case, we expect all $q_{k}$ to have nonzero projection on $R_{1}$. Hence, they will all scale as $\zeta^{1}$, giving

$$
q_{k}(\tau) \sim \zeta^{1}(\tau) \sim \epsilon^{1 /(\rho-1)} .
$$

Threshold-linear transfer function.--In Sec. V, we have shown that in one population with a threshold-linear transfer function $(\nu=1)$, the excess mean input is zero at the transition (Fig. 2). Our numerical solutions show that in two-population networks with this transfer function, $x_{l}$ are no longer exactly zero at the transition; nevertheless, they remain small in a wide region above the transition. Assuming $\left|x_{l}\right| \ll 1$, the stability matrix can be written as

$$
\tilde{M}_{k l}=\tilde{g}_{k l}^{2} H\left(-x_{l}\right) \approx 0.5 \tilde{g}_{k l}^{2} .
$$

The mean field expression for the variance, Eq. (12), can be written as $1=\sum_{l} \tilde{g}_{k l}^{2}\left\langle\phi^{2}\left(z+x_{l}\right)\right\rangle_{z} \approx 0.5 \sum_{l} \tilde{g}_{k l}^{2}$. Hence, we find that $\sum_{l} \tilde{M}_{k l}=1+\mathcal{O}\left(x_{l}\right), \forall k$, implying that the eigenvector corresponding to the largest eigenvalue of $\tilde{\mathbf{M}}$ is uniform to leading order in $x_{k}$. It follows that near criticality, when $\left|x_{k}\right|$ is small, $R^{1}$ is approximately uniform, and all $q_{k}$ are nearly equal, as can be seen in Fig. 10. Furthermore, if $x_{k}$ remain small away from criticality, then $R^{1}$ is nearly uniform, which can explain the numerical results observed by Ref. [37]. It should be interesting to see further analysis of the exact solutions for $x_{k}$.

[1] M. Abeles, Corticonics: Neural Circuits of the Cerebral Cortex (Cambridge University Press, Cambridge, England, 1991).

[2] W. Bair, C. Koch, W. Newsome, and K. Britten, Power Spectrum Analysis of Bursting Cells in Area MT in the Behaving Monkey, J. Neurosci. 14, 2870 (1994).

[3] W. R. Softky and C. Koch, The Highly Irregular Firing of Cortical Cells Is Inconsistent with Temporal Integration of Random EPSPs, J. Neurosci. 13, 334 (1993).

[4] G. R. Holt, W. R. Softky, C. Koch, and R. J. Douglas, A Comparison of Discharge Variability In Vitro and In Vivo in Cat Visual Cortex Neurons, J. Neurophysiol. 75, 1806 (1996). 
[5] K. Kang, R. M. Shapely, and H. Sompolinsky, Information Tuning of Populations of Neurons in Primary Visual Cortex, J. Neurosci. 24, 3726 (2004).

[6] H. Sompolinsky, A. Crisanti, and H. J. Sommers, Chaos in Randon Neural Networks, Phys. Rev. Lett. 61, 259 (1988).

[7] C. van Vreeswijk and H. Sompolinsky, Chaos in Neuronal Networks with Balanced Excitatory and Inhibitory Activity, Science 274, 1724 (1996).

[8] C. van Vreeswijk and H. Sompolinsky, Chaotic Balanced State in a Model of Cortical Circuits, Neural Comput. 10, 1321 (1998).

[9] A. Renart, J. de la Rocha, P. Bartho, L. Hollender, N. Parga, A. Reyes, and K. D. Harris, The Asynchronous State in Cortical Circuits, Science 327, 587 (2010).

[10] N. Brunel, Phase Diagrams of Sparsely Connected Networks of Excitatory and Inhibitory Spiking Neurons, Neurocomputing; Variable Star Bulletin 32-33, 307 (2000).

[11] L. Busing, B. Schrauwen, and R. Legenstein, Connectivity, Dynamics, and Memory in Reservoir Computing with Binary and Analog Neurons, Neural Comput. 22, 1272 (2010).

[12] M. Lukoševičius and H. Jaeger, Reservoir Computing Approaches to Recurrent Neural Network Training, Comput. Sci. Rev. 3, 127 (2009).

[13] D. Sussillo and L. F. Abbott, Generating Coherent Patterns of Activity from Chaotic Neural Networks, Neuron 63, 544 (2009).

[14] O. Barak, D. Sussillo, R. Romo, M. Tsodyks, and L. F. Abbott, From Fixed Points to Chaos: Three Models of Delayed Discrimination, Prog. Neurobiol. 103, 214 (2013).

[15] R. Legenstein and W. Maass, Edge of Chaos and Prediction of Computational Performance for Neural Circuit Models, Neural Netw. 20, 323 (2007).

[16] W. Maass, T. Natschläger, and H. Markram, Real-Time Computing without Stable States: A New Framework for Neural Computation Based on Perturbations, Neural Comput. 14, 2531 (2002).

[17] H. Jaeger and H. Haas, Harnessing Nonlinearity: Predicting Chaotic Systems and Saving Energy in Wireless Communication, Science 304, 78 (2004).

[18] T. Toyoizumi and L. F. Abbott, Beyond the Edge of Chaos: Amplification and Temporal Integration by Recurrent Networks in the Chaotic Regime, Phys. Rev. E 84, 051908 (2011).

[19] N. Bertschinger and T. Natschlager, Real-Time Computation at the Edge of Chaos in Recurrent Neural Networks, Neural Comput. 16, 1413 (2004).

[20] A. M. Saxe, J. L. McClelland, and S. Ganguli, Exact solutions to the nonlinear dynamics of learning in deep linear neural networks, arXiv:1312.6120.

[21] A. Graves, A.-R. Mohamed, and G. Hinton, Speech Recognition with Deep Recurrent Neural Networks, in ICASSP 2013-IEEE International Conference on Acoustics, Speech and Signal Processing (ICASSP) (IEEE, 2013) pp. 6645-6649.

[22] G. Mesnil, Y. Dauphin, K. Yao, Y. Bengio, L. Deng, D. Hakkani-Tur, X. He, L. Heck, G. Tur, D. Yu, and G. Zweig, Using Recurrent Neural Networks for Slot Filling in Spoken Language Understanding, IEEE/ACM Transactions on Audio, Speech, and Language Processing 23, 530 (2015).
[23] R. Pascanu, C. Gulcehre, K. Cho, and Y. Bengio, How to Construct Deep Recurrent Neural Networks, arXiv:1312.6026.

[24] N. Brunel, Dynamics of Sparsely Connected Networks of Excitatory and Inhibitory Spiking Neurons, J. Comput. Neurosci. 8, 183 (2000).

[25] S. Ostojic, Two Types of Asynchronous Activity in Networks of Excitatory and Inhibitory Spiking Neurons, Nat. Neurosci. 17, 594 (2014).

[26] M. Helias, T. Tetzlaff, and M. Diesmann, The Correlation Structure of Local Neuronal Networks Intrinsically Results from Recurrent Dynamics, PLoS Comput. Biol. 10, e1003428 (2014).

[27] K. H. Fischer and J. A. Hertz, Spin Glasses (Cambridge University Press, Cambridge, England, 1991).

[28] M. Mezard, G. Parisi, and M. A. Virasoro, Spin Glass Theory and Beyond, Lecture Notes in Physics Series (World Scientific, Singapore, 1987).

[29] C. van Vreeswijk and H. Sompolinsky, Irregular Activity in Large Networks of Neurons, in Methods and Models in Neurophysics, Volume LXXX: Lecture Notes of the Les Houches Summer School, edited by C. Chow, B. Gutkin, D. Hansel, C. Meunier, and J. Dalibard (Elsevier, Amsterdam, 2005), Chap. 9, pp. 341-402.

[30] T. Cabana and J. Touboul, Large Deviations, Dynamics and Phase Transitions in Large Stochastic and Disordered Neural Networks, J. Stat. Phys. 153, 211 (2013).

[31] O. Faugeras, J. Touboul, and B. Cessac, A Constructive Mean-Field Analysis of Multi Population Neural Networks with Random Synaptic Weights, in Front. Syst. Neurosci. Conference Abstract: Computational and Systems Neuroscience (2009).

[32] H. Sompolinsky and A. Zippelius, Relaxational Dynamics of the Edwards-Anderson Model and the Mean-Field Theory of Spin-Glasses, Phys. Rev. B 25, 6860 (1982).

[33] M. A. Buice, J. D. Cowan, and C. C. Chow, Systematic Fluctuation Expansion for Neural Network Activity Equations, Neural Comput. 22, 377 (2010).

[34] N. J. Priebe and D. Ferster, Inhibition, Spike Threshold, and Stimulus Selectivity in Primary Visual Cortex, Neuron 57, 482 (2008).

[35] D. Hansel and C. Van Vreeswijk, How Noise Contributes to Contrast Invariance of Orientation Tuning in Cat Visual Cortex, J. Neurosci. 22, 51185 (2002).

[36] A. Wolf, J. B. Swift, H. L. Swinney, and J. A. Vastan, Determining Lyapunov Exponents from a Time Series, Physica (Amsterdam) 16D, 285 (1985).

[37] J. Aljadeff, M. Stern, and T. Sharpee, Transition to Chaos in Random Networks with Cell-Type-Specific Connectivity, Phys. Rev. Lett. 114, 088101 (2015).

[38] G. Wainrib and J. Touboul, Topological and Dynamical Complexity of Random Neural Networks, Phys. Rev. Lett. 110, 118101 (2013).

[39] D. Hansel and H. Sompolinsky, Chaos and Synchrony in a Model of a Hypercolumn in Visual Cortex, J. Comput. Neurosci. 3, 7 (1996).

[40] P. Greengard, The Neurobiology of Slow Synaptic Transmission, Science 294, 1024 (2001).

[41] I. Ginzburg and H. Sompolinsky, Theory of Correlations in Stochastic Neural Networks, Phys. Rev. E 50, 3171 (1994). 
[42] Yashar Ahmadian, Francesco Fumarola, and Kenneth D. Miller, Properties of Networks with Partially Structured and Partially Random Connectivity, Phys. Rev. E 91, 012820 (2015).

[43] A. Litwin-Kumar and B. Doiron, Slow Dynamics and High Variability in Balanced Cortical Networks with Clustered Connections, Nat. Neurosci. 15, 1498 (2012).

[44] P. Dayan and L.F. Abbott, Theoretical Neuroscience: Computational and Mathematical Modeling of Neural Systems, Computational Neuroscience (Massachusetts Institute of Technology Press, Cambridge, MA, 2005).

[45] T. W. Troyer, A. E. Krukowski, N. J. Priebe, and K. D. Miller, Contrast-Invariant Orientation Tuning in Cat Visual Cortex: Thalamocortical Input Tuning and Correlation-Based Intracortical Connectivity, J. Neurosci. 18, 5908 (1998).

[46] D. J. Heeger, Normalization of Cell Responses in Cat Striate Cortex, Visual neuroscience 9, 181 (1992).

[47] H. Sompolinsky and D. Hansel, Modeling Feature Selectivity in Local Cortical Circuits, in Methods in Neuronal Modeling: From Synapse to Networks, edited by C. Koch and I. Segev, 2nd ed. (MIT Press, Cambridge, MA, 1998), Chap. 13.

[48] D. G. Albrecht, Visual Cortex Neurons in Monkey and Cat: Effect of Contrast on the Spatial and Temporal Phase Transfer Functions, Visual neuroscience 12, 1191 (1995).

[49] H. C. Tuckwell, Introduction to Theoretical Neurobiology, Cambridge Books Online (Cambridge University Press, Cambridge, England, 1988), Vol. 1.

[50] N. Brunel, V. Hakim, and M. J.E. Richardson, Single Neuron Dynamics and Computation, Curr. Opin. Neurobiol. 25, 149 (2014).

[51] J. M. Rinzel and G. B. Ermentrout, Analysis of Neuronal Excitability, in Methods in Neuronal Modeling, edited by C. Koch and I. Segev (MIT Press, Cambridge, MA, 1989), pp. 135-170.

[52] B. Ermentrout, Linearization of $f-I$ Curves by Adaptation, Neural Comput. 10, 1721 (1998).
[53] O. Shriki, D. Hansel, and H. Sompolinsky, Rate Models for Conductance-Based Cortical Neuronal Networks, Neural Comput. 15, 1809 (2003).

[54] K. Rajan, L. F. Abbott, and H. Sompolinsky, StimulusDependent Suppression of Chaos in Recurrent Neural Networks, Phys. Rev. E 82, 011903 (2010).

[55] B. Ermentrout, Reduction of Conductance-Based Models with Slow Synapses to Neural Nets, Neural Comput. 6, 679 (1994).

[56] E. Brown, R. Barbieri, V. Ventura, R. Kass, and L. Frank, The Time-Rescaling Theorem and Its Application to Neural Spike Train Data Analysis, Neural Comput. 14, 325 (2002).

[57] R. Zillmer, N. Brunel, and D. Hansel, Very Long Transients, Irregular Firing, and Chaotic Dynamics in Networks of Randomly Connected Inhibitory Integrate-and-Fire Neurons, Phys. Rev. E 79, 031909 (2009).

[58] S. Jahnke, R.-M. Memmesheimer, and M. Timme, Stable Irregular Dynamics in Complex Neural Networks, Phys. Rev. Lett. 100, 048102 (2008).

[59] A. Zumdieck, M. Timme, T. Geisel, and F. Wolf, Long Chaotic Transients in Complex Networks, Phys. Rev. Lett. 93, 244103 (2004).

[60] M. Monteforte and F. Wolf, Single Cell Dynamics Determine Strength of Chaos in Collective Network Dynamics, BMC Neurosci. 12, P225 (2011).

[61] S. Ostojic and N. Brunel, From Spiking Neuron Models to Linear-Nonlinear Models, PLoS Comput. Biol. 7, e1001056 (2011).

[62] E.S. Schaffer, S. Ostojic, and L. F. Abbott, A ComplexValued Firing-Rate Model that Approximates the Dynamics of Spiking Networks, PLoS Comput. Biol. 9, e1003301 (2013).

[63] V. Mante, D. Sussillo, K. V. Shenoy, and W. T. Newsome, Context-Dependent Computation by Recurrent Dynamics in Prefrontal Cortex, Nature (London) 503, 78 (2013).

[64] O. Harish and D. Hansel, Asynchronous Rate Chaos in Spiking Neuronal Circuits, PLoS Comput. Biol. 11, e1004266 (2015). 